\title{
INFLUENCE DE LA NATURE DU PÂTURAGE SUR LA PRODUCTION LAITIÈRE ET LA COMPOSITION DU LAIT
}

\author{
C. DEMARQUILLY \\ avec la collaboration technique de Marie-Claire Engrand. J-F. Cristofini, Y. Manis, \\ 3. Marquis et J-C. Turc. \\ Station de Recherches sur l'Élevage des Ruminants, \\ Centre national de Recherches zootechniques, Jouy-en-Josas (Seine-et-Oise)
}

\section{SOMMAIRE}

Les espèces et variétés fourragères actuellement utilisées pour les prairies temporaires n'ont pas fait l'objet d'étude systématique quant à leurs influences possibles sur la production animale. Nous rapportons ici une série d'observations effectuées pendant 4 années consécutives, et dans deux stations, sur l'influence de la nature du pâturage sur la production et la composition du lait. Pour cela, chaque jour, nous avons mesuré la production laitière et déterminé le taux butyreux d'un certain nombre de vaches pâturant ou recevant en stabulation de l'herbe fauchée.

Nous avons constaté certaines actions nettes et reproductibles : lorsque les vaches ont consommé de l'herbe à base de Dactyle ou de Luzerne, la production laitière a diminué et le taux butyreux a augmenté. Inversement, la production laitière a augmenté et le taux butyreux a diminué quand les vaches ont consommé de l'herbe à base de Ray-grass, de Fétuque des prés, de Fléole ou de Trèfle blanc. Ces variations du taux butyreux suivant la nature de l'herbe consommée n'avaient, à notre connaissance, jamais été mises en évidence.

Dans l'état actuel de nos connaissances, il semble que les variations du niveau des apports énergétiques et les modifications des fermentations du rumen, avec la nature du pâturage, puissent rendre compte respectivement de ces variations de la production de lait et de matières grasses et de celles du taux butyreux. Cepenc'ant, il ne faut pas exclure l'intervention de facteurs stimulants ou inhibiteurs.

\section{INTRODUCTION}

La pâturage des prairies temporaires a pris beaucoup d'importance en France depuis une dizaine d'années. L'agriculteur dispose maintenant de nombreuses espèces et variétés, qui ont été sélectionnées en fonction de leurs caractères de production, et de leur résistance aux maladies ou aux conditions climatiques défavorables. Malheureusement, on n'a pas pu tenir compte dans cette sélection, de la valeur alimentaire de ces plantes pour l'animal, dont la réponse est primordiale.

A l'étranger, plusieurs auteurs cependant ont constaté des variations de la pro- 
duction laitière des vaches, en fonction de la nature des prairies temporaires pâturées. CASTLE (I953) en Angleterre a constaté que le Ray-grass a permis chaque année, une production laitière plus élevée que le Dactyle, à la fois par vache et par hectare, bien que ces deux variétés aient produit dans les conditions de son essai, une égale quantité de matière sèche à l'hectare. CASTLE en a conclu que "le Dactyle semble manquer d'une qualité indéfinissable pour la production laitière, qualité que le Ray-grass possède ". PATERson (1956, I959) a constaté une diminution de la production laitière quand les vaches pâturaient du Dactyle, " le Dactỵle, si bien contrôlé soit-il, nous a donné, dit-il, une décroissance excessive de la production laitière, à moins qu'il ne soit mélangé à d'autres graminées. "Toujours en Angleterre, Ivins (I959) a mis, lui aussi, en évidence une production laitière par vache. et par hectare plus faible avec le Dactyle qu'avec le Ray-grass anglais ou le mélange Fétuque des prés-Fléole. En Irlande, Boyd et JEnnings (I960), ont montré que les prairies à base de Dactyle entraînaient une diminution de la production laitière, et en ont conclu que "l'emploi des prairies de Dactyle pour les vaches laitières dans ce pays, ne se justifie que dans les endroits au sol sec, peu profond, qui normalement " rôtissent " en été.

En revanche, il n'y a pas eu, à notre connaissance, d'études mettant en évidence des variations de la composition du lait, en particulier du taux butyreux, suivant la nature du pâturage.

L'objet de la présente étude a été :

Io) d'enregistrer au cours de plusieurs années successives, les variations de la production laitière individuelle et de la teneur en matières grasses du lait, provoquées par les changements de la nature du pâturage à l'intérieur de "chaînes " de pâturage.

$\left.2^{\circ}\right)$ d'analyser certains facteurs de ces variations: quantité d'éléments nutritifs consommés, modifications des fermentations du rumen.

Aussi durant quatre années consécutives, de 1958 à I96r, nous avons enregistré la production laitière journalière et la teneur journalière en matières grasses du lait d'un certain nombre de vaches choisies dans les deux troupeaux des stations de JouY et de LA MrNik̀nE. Ces vaches pâturaient avec le reste du troupeau des "chaînes" de pâturage ou recevaient en stabulation de l'herbe fauchée dans les prairies pâturées par le troupeau. Nous avons mesuré les quantités de matière sèche d'herbe consommées par les vaches alimentées en stabulation, et en rg6o et ig6r déterminé la digestibilité de 1'herbe employée. En outre, en I96I, nous avons analysé des échantillons de jus de rumen prélevé par tubage tant sur les vaches au pâturage qu'en stabulation pour déterminer la concentration et la composition du mélange des acides gras volatils.

Les premiers résultats de cette étude ont déjà été rapportés (JARRIGE et JOURNET I959).

\section{MÉTHODES ETT TECHNIQUES}

\section{Animaux}

Dans le troupeau de chacune des deux stations de Jouy et de La Minière distantes de Io $\mathrm{km}$, nous avons choisi chaque année de I 5 à 25 vaches, suivant les disponibilités en main-d'œuvre pour effectuer les mesures et contrôles nécessaires.

Les vaches utilisées étaient en proportion à peu près égale de race française frisonne pie-noire 
et normande. Elles étaient, lors de la mise à l'herbe, dans le début de la phase décroissante de la lactation de façon à pouvoir être suivies durant la totalité ou la majeure partie de la saison de pâturage. Nous connaissions la ou les productions laitières antérieures; elles avaient été bonnes et avaient eu une évolution normale.

L'étude a porté d'une part sur des vaches au pâturage (au total 69), d'autre part sur des vaches en stabulation recevant de l'herbe coupée (au total 24 ). Le tableau i en donne la répartition suivant les années, les stations et les essais.

TABLEAU I

Répartition des animaux suivant les années, les stations et les essais

\begin{tabular}{|c|c|c|c|c|}
\hline Année & Étable & $\begin{array}{l}\text { Nombre de vaches } \\
\text { contrôlées }\end{array}$ & $\begin{array}{c}\text { Mode } \\
\text { d'alimentation }\end{array}$ & $\begin{array}{c}\text { Périodes } \\
\text { d'observations }\end{array}$ \\
\hline 1958 & $\begin{array}{l}\text { Jouy } \\
\text { La Minière }\end{array}$ & $\begin{array}{l}12 \\
19\end{array}$ & $\begin{array}{l}\text { pâturage } \\
\text { pâturage }\end{array}$ & $\begin{array}{rll}26 / 5 & \text { au } & 17 / 7 \\
1 / 6 & \text { au } & 13 / 10\end{array}$ \\
\hline 1959 & $\begin{array}{c}\text { Jouy } \\
\text { La Minière }\end{array}$ & $12\left\{\begin{array}{l}6 \\
6\end{array}\right.$ & $\begin{array}{l}\text { Iâturage } \\
\text { stabulation } \\
\text { pâturage }\end{array}$ & $\begin{array}{l}29 / 4 \text { au } 5 / 7 \\
11 / 6 \text { au } 31 / 7\end{array}$ \\
\hline 1960 & Jouy & $16\left\{\begin{array}{l}8 \\
8\end{array}\right.$ & $\begin{array}{l}\text { pâturage } \\
\text { stabulation }\end{array}$ & $\begin{array}{l}30 / 5 \text { au } 27 / 7 \\
30 / 5 \text { au } 30 / 7\end{array}$ \\
\hline 1961 & La Minière & 果) $\begin{array}{l}10 \\
10\end{array}$ & $\begin{array}{l}\text { pâturage } \\
\text { stabulation }\end{array}$ & $\begin{array}{l}15 / 5 \text { au } 22 / 10 \\
15 / 5 \text { au } 1 / 9\end{array}$ \\
\hline
\end{tabular}

\section{Alimentation el mode de vie}

Les vaches au pâturage ont consommé l'herbe des différentes prairies en pâturage rationné. La clôture électrique était avancée deux fois par jour à $8 \mathrm{~h}$ et $18 \mathrm{~h}$ et les vaches ne rentraient à l'étable que pour la traite du matin et du soir. Excepté à la fin du premier cycle de végétation, l'herbe était au stade feuillu et âgée de 4 à 7 semaines. Les vaches ont reçu en complément une ration d'aliments concentrés à raison de $0,5 \mathrm{~kg}$ par $\mathrm{kg}$ de lait $4 \mathrm{p}$. I 00 produit au-dessus d'une production de I I à I $6 \mathrm{~kg}$ de lait 4 p. 1oo, suivant le poids des vaches et l'âge de l'herbe. La quantité d'aliments concentrés distribuée était ajustée chaque semaine en fonction de la quantité de lait produite la semaine précédente et de son évolution.

Les vaches en stabulation ont reçu de l'herbe verte à volonté (ro à ${ }_{5}$ p. roo de refus) en 4 repas par jour, à $5 \mathrm{~h}, 9 \mathrm{~h}, \mathrm{I} 5 \mathrm{~h}$ et $\mathrm{I} 8 \mathrm{~h}$. L'herbe nécessaire était fauchée chaque matin dans une bande réservée à la fauche, délimitée à l'intérieur des parcelles pâturées par le reste du troupeau. Les vaches ont reçu, en complément, une ration d'aliments concentrés calculée suivant leur production laitière, leur poids et les quantités de matière sèche d'herbe qu'elles consommaient. La quantité d'aliments concentrés distribuée était ajustée chaque semaine, en fonction des productions et des consommations enregistrées la semaine précédente.

\section{Prairies}

Les prairies temporaires utilisées comprenaient soit une seule graminée (à l'exception de la Fétuque des prés semée parfois en mélange avec de la Fléole) associée à une légumineuse, soit de la Luzerne pure. Le semis avait eu lieu sur sol nu au printemps et les prairies ont été exploitées 3 à 6 ans avant d'être retournées. Elles ont reçu une fumure phospho-potassique chaque hiver (rookg environ de $\mathrm{P}$ et $\mathrm{K}$ ) ; les prairies à base de graminées ont de plus reçu $40 \mathrm{~kg}$ d'azote au printemps avant le départ de la végétation et deux ou trois épandages de $40 \mathrm{~kg}$ d'azote lors des exploitations ultérieures. Celles-ci ont eu lieu toutes les 4 ia 7 semaines suivant les saisons et les conditions climati- 
C. DEMARQUILIY

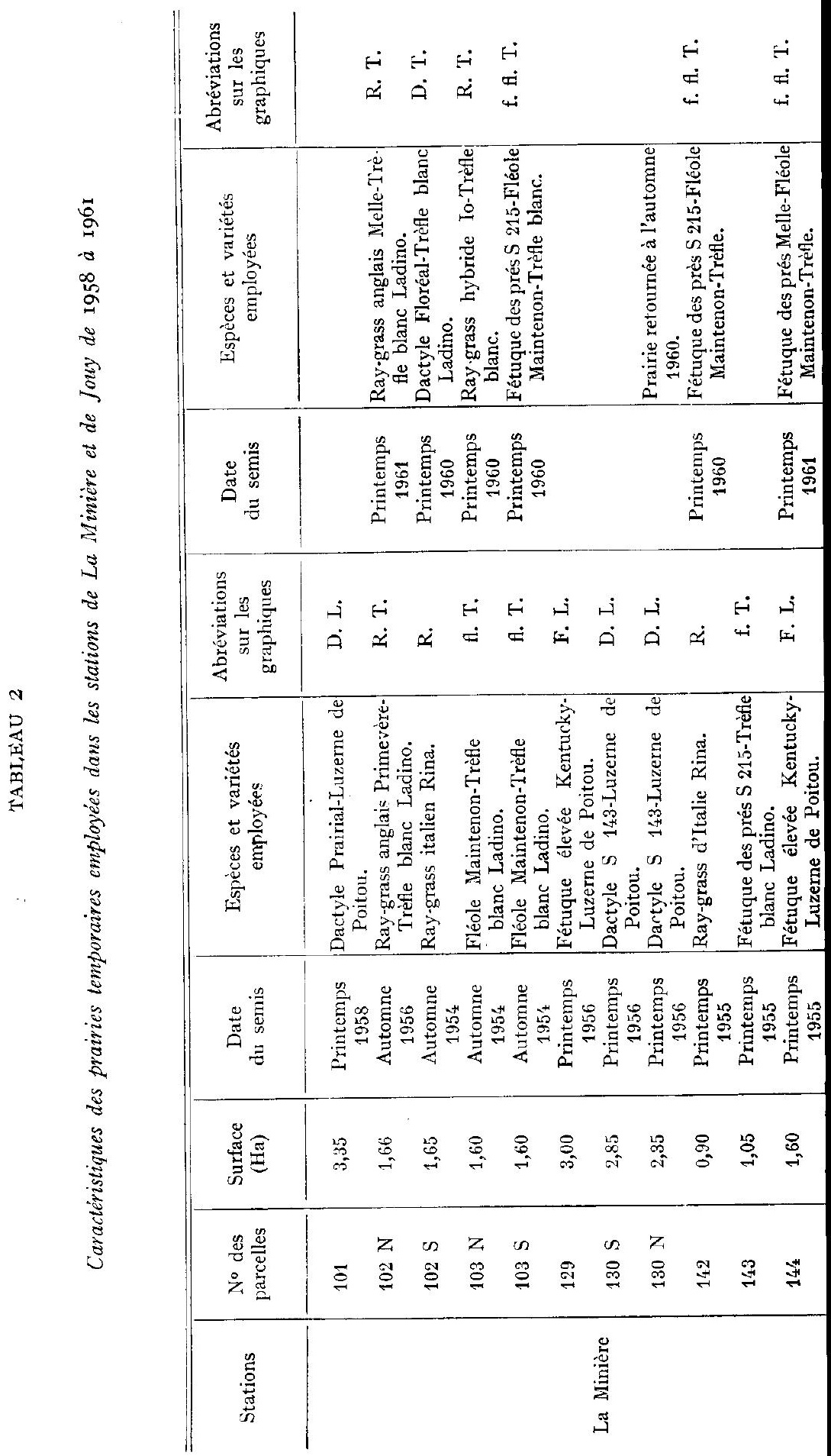




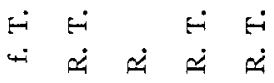

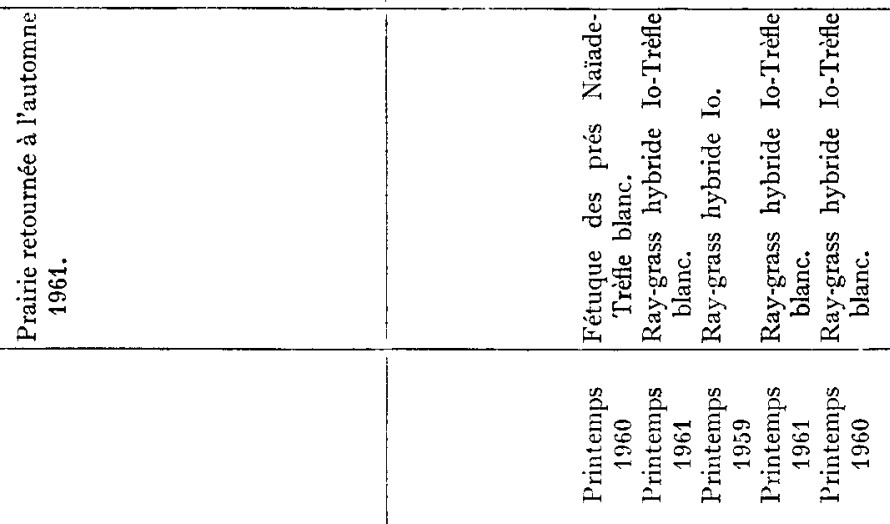

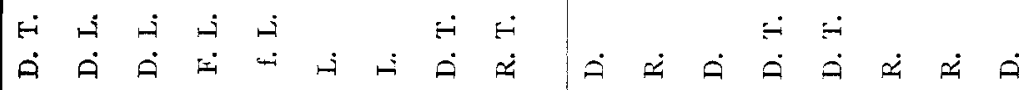

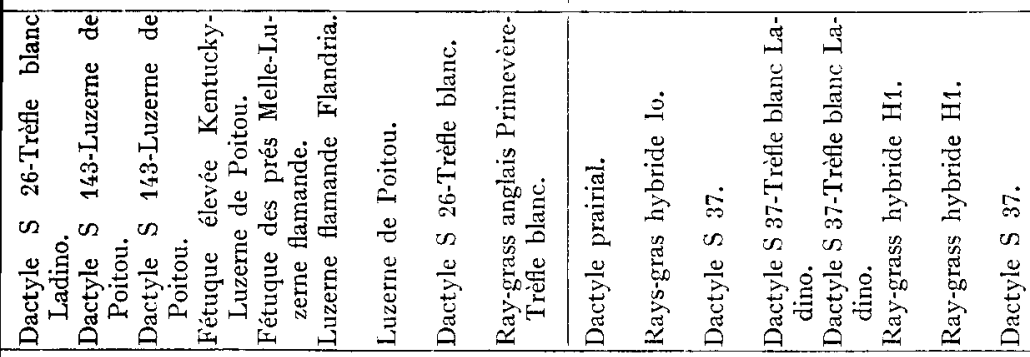

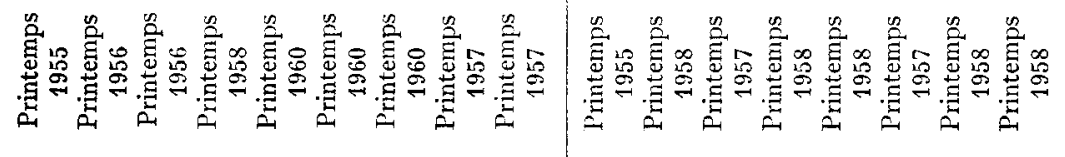

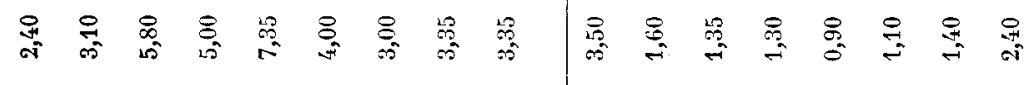

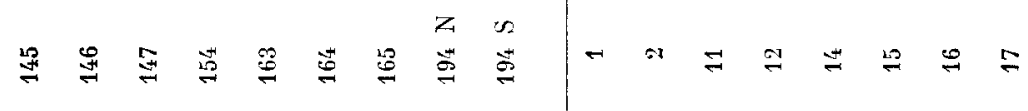


ques. Les refus laissés par les animaux ont été fauchés après la première et la deuxième exploitation.

Le tableau 2 donne les espèces et variétés utilisées au cours des 4 années dans les deux stations. Le Trèfle blanc a toujours disparu rapidement des prairies de Jouy, mais prenait le dessus sur les graminées autres que les dactyles dans les prairies temporaires anciennes de $\mathrm{La}$ Minière après le premier cycle de végétation; c'était le cas notamment en $195^{8}$. De plus, à Jouy, certaines prairies de Dactyle étaient progressivement envahies par de la Houlque laineuse.

\section{TABLEAU 3}

Relevé météorologique des années $195^{88}$ à $196 \mathrm{I}$ à La Minière

\begin{tabular}{|c|c|c|c|c|}
\hline & Mois & $\begin{array}{l}\text { Température } \\
\text { moyenne } \\
\text { (degré) }\end{array}$ & $\begin{array}{c}\text { Durée } \\
\text { d'ensoleillement } \\
\text { (heure) }\end{array}$ & $\begin{array}{l}\text { Pluie } \\
\text { (mm) }\end{array}$ \\
\hline 1958 & 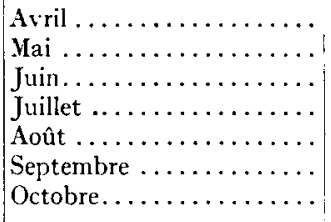 & $\begin{array}{r}9,8 \\
16,1 \\
17,9 \\
20,9 \\
19,1 \\
23,0 \\
11,9\end{array}$ & $\begin{array}{r}158,10 \\
173,10 \\
178,40 \\
202,50 \\
127,40 \\
153.20 \\
94,00\end{array}$ & $\begin{array}{l}52,8 \\
55,4 \\
73,9 \\
73,4 \\
61,0 \\
32.7 \\
72,7\end{array}$ \\
\hline 1959 & 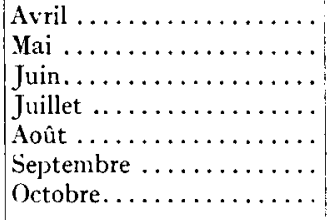 & $\begin{array}{r}9,8 \\
13,5 \\
16,9 \\
20,5 \\
18,7 \\
17.7 \\
12,2\end{array}$ & $\begin{array}{l}157,30 \\
229,30 \\
279,00 \\
333,30 \\
218,40 \\
195.30 \\
172,30\end{array}$ & $\begin{array}{r}79,3 \\
32,0 \\
37,0 \\
13,5 \\
33,7 \\
3,9 \\
62,4\end{array}$ \\
\hline 1960 & 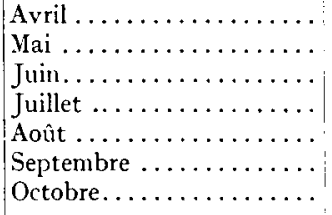 & $\begin{array}{r}9.4 \\
15,0 \\
17 . ; \\
15,4 \\
16,5 \\
12 . ; \\
11, ;\end{array}$ & $\begin{array}{r}105,10 \\
189,20 \\
115,10 \\
108,00 \\
1,10,20 \\
90,50 \\
35,10\end{array}$ & $\begin{array}{r}1,4,4 \\
19,8 \\
79,0 \\
79,0 \\
112,6 \\
74,2 \\
121,2\end{array}$ \\
\hline 1961 & 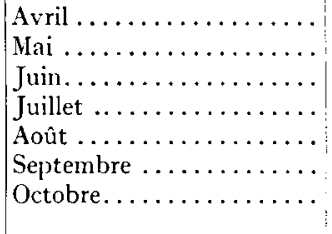 & $\begin{array}{l}11,9 \\
11,8 \\
15,7 \\
16,9 \\
17,5 \\
18.2 \\
11,1\end{array}$ & $\begin{array}{l}112,70 \\
200,30 \\
194,20 \\
185,50 \\
200,50 \\
162,20 \\
101,10\end{array}$ & $\begin{array}{r}46,5 \\
33,4 \\
58,0 \\
53,5 \\
4,1 \\
70,2 \\
70,0\end{array}$ \\
\hline
\end{tabular}

Pour alimenter les troupeaux de 40 vaches laitières à Jouy et de 80 vaches laitières à La Minière nous avons disposé respectivement de 15 et de 25 ha de prairies temporaires. Ces superficies étaient insuffisantes pour offrir aux vaches de l'herbe à volonté durant les périodes de sécheresse. Durant ces périodes les expériences ont été interrompues et les vaches ont reçu une alimentation complémentaire (drèches, ensilage...).

Les essais se sont déroulés sous des conditions climatiques très différentes (tableau 3 ); en particulier l'année 1959 a été très sèche et l'année I 960 très humide. 


\section{Mesures}

Nous avons laissé de côté dans cette étude les cinq premières semaines après la mise à l'herbe car celle-ci entraîne des variations de la production laitière et de la composition du lait qui semblent indépendantes de la nature de l'herbe (DEMARQUILly et JOURNET, 1962).

Le lait de chaque vache a été pesé à chaque traite et un échantillon de lait a été prélevé. $\mathrm{A}$ partir de ces prélèvements il a été constitué un échantillon individuel pondéré du lait produit en 24 heures dont la teneur en matières grasses a été déterminée par la méthode Gerber.

Les quantités individuelles de matière verte et de matière sèche d'herbe consommée par chacune des vaches en stabulation ont été mesurées par pesée des quantités distribuées et refusées et détermination de la teneur en matière sèche de l'herbe et des refus de chaque repas.

En 1960 et I96I, le coefficient de digestibilité de l'herbe des différentes prairies a été mesuré sur un lot de 5 béliers alimentés ad libitum ( 5 à I 5 p. Ioo de refus). Les béliers ont reçu chaque jour, en trois repas, de l'herbe fauchée le matin pour alimenter les vaches en stabulation. Les périodes de mesure ont correspondu aux différents séjours des vaches sur les prairies; les périodes préexpérimentales ont été réduites à 2 ou 3 jours, et les périodes de mesure ont duré au minimum 5 jours. Les échantillons représentatifs d'herbe et de fèces correspondant à chaque période ont été analysés pour mesurer leurs teneurs en matière organique, en matières azotées et en cellulose brute. La valeur énergétique de l'herbe a été exprimée en unités fourragères (U.F.) par $\mathrm{kg}$ de matière sèche, selon la méthode proposée par BREIREM (1954) :

$$
\mathrm{UF} / \mathrm{kg} \mathrm{MS}=\frac{2,36 \text { matière organique digestible }-1,20 \text { matière organique indigestible }}{165^{\circ}}
$$

En 1961, du jus de rumen a été prélevé par tubage à trois vaches en stabulation et trois vaches au pâturage, en huit occasions sur différentes variétés d'herbe (tableau ${ }_{5}$ ). Ce jus a été amené à $\mathrm{pH} 9$, bloqué avec I p. 100 de $\mathrm{NO}_{3} \mathrm{Hg}$ I p. Ioo et conservé jusqu'à l'analyse en chambre froide à $-5^{\circ} \mathrm{C}$ dans des flacons de polyéthylène. Les teneurs en acide acétique, propionique et butyrique ont été déterminées sur les échantillons individuels ou pondérés par période et par lot par chromatographie en phase gazeuse (JAMES et MARTIN, 195I) après distillation (FriedemanN, I938) et évaporation (ElSDEN modifié par ZELTER, I958).

\section{Problèmes posés par l'interprétation des résultats}

Les temps de séjour sur les prairies ont été extrêmement variables suivant la superficie des prairies et les différentes exploitations. Aussi avons-nous parfois été obligés de grouper certaines prairies de variétés et même d'espèces différentes, après avoir vérifié que la production laitière et le taux butyreux n'avaient pas varié lorsque les vaches avaient pâturé ces prairies. C'est ainsi que nous avons parfois groupé des prairies de Ray-grass anglais, de Ray-grass italien, de Fléole et de Fétuque des prés d'une part, de Dactyle et de Dactyle-Luzerne d'autre part.

Nous avons été ensuite amenés à chercher quelle était la meilleure méthode permettant de caractériser l'influence de la nature du pâturage sur la production laitière et le taux butyreux du lait.

Cette influence peut être d'abord mesurée par les différences entre les valeurs moyennes (par jour et par vache) observées au cours du séjour sur deux prairies pâturées successivement, cela pour le lait, le taux butyreux et la matière grasse. Cette méthode présente deux inconvénients :

- le temps de séjour sur deux prairies successives a presque toujours été inégal,

- l'influence du stade de lactation peut devenir importante si les temps de séjour sont longs.

Cette dernière est faible pour le taux butyreux qui évolue lentement avec le stade de lactation. Il n'en est pas de même pour la production laitière qui diminue en moyenne de ıo p. ıoo par mois ; lorsque la production laitière diminue en passant d'une prairie à la suivante, nous ne savons pas quelle est la part exacte de cette diminution qui est due au stade de lactation. Aussi dans les tableaux $4,5,7,8,9$, IO, II, I2, I 3 , avons-nous indiqué la diminution théorique de la production laitière moyenne par vache qui est due à l'évolution de la lactation durant le séjour sur la prairie.

Une autre méthode nous permet d'éliminer ces deux inconvénients. Elle consiste à comparer les moyennes journalières de la production laitière et du taux butyreux des 2 ou 3 derniers jours sur une prairie aux moyennes des 2 ou 3 premiers jours sur la prairie suivante. Malheureusement une moyenne effectuée sur 2 ou 3 jours seulement est beaucoup trop soumise aux fluctuations journalières qui sont très importantes en particulier pour le taux butyreux. En revanche, elle nous 
permet de mesurer la rapidité et l'ampleur de la réponse au changement de nature du pâturage, de la quantité et de la composition du lait.

Aucune de ces deux méthodes n'est parfaite mais elles se complètent l'une l'autre : nous les utiliserons toutes les deux pour la présentation des résultats.

\section{RÉSULTATS}

Pour chaque année les résultats ont été résumés par :

- un graphique montrant l'évolution des valeurs journalières de la production laitière, du taux butyreux et de la production de matières grasses, sur les différentes prairies pâturées par le lot expérimental, cela durant les périodes où aucune alimentation complémentaire autre que l'aliment concentré n'était distribuée.

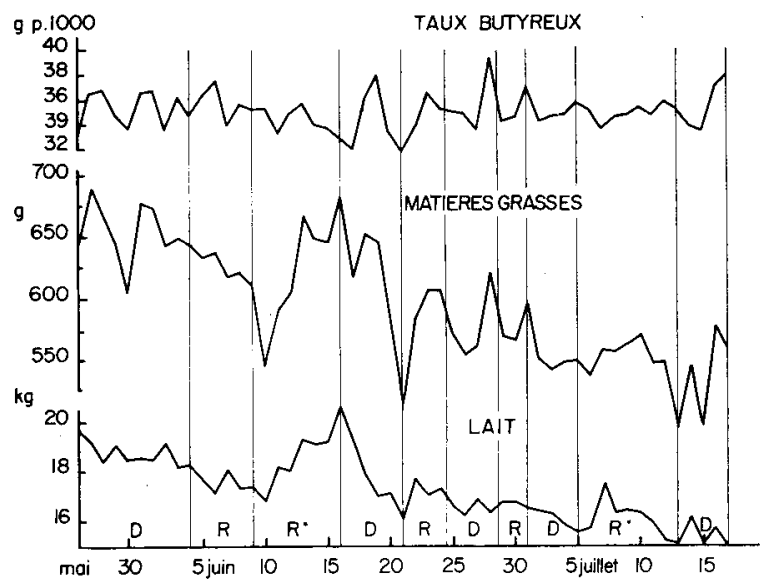

FIG. I. - Infuence de la nature du pâturage sur l'évolution des productions journalières de lait et de matières grasses et sur le taux butyreux (moyenne de i 2 vaches au pâturage - Jouy, 1958).

$$
\begin{aligned}
& \mathrm{D} \quad \text { : Dactyle ; } \\
& \mathrm{R} \text { : Ray-grass ; } \\
& \mathrm{R}^{*} \text { : Ray-grass exploité l'année du semis. }
\end{aligned}
$$

- un tableau donnant durant les mêmes périodes les différences entre :

- les moyennes journalières, sur deux prairies pâturées successivement, de la production laitière, du taux butyreux et de la production de matières grasses.

- les moyennes journalières des deux derniers jours sur une prairie et des deux premiers jours sur la prairie suivante pour le lait, le taux butyreux et les matières grasses.

\section{JoUy 1958}

Le lot expérimental de $\mathrm{I} 2$ vaches a pâturé avec le reste du troupeau des prairies de Dactyle et de Ray-grass anglais presque pur; une des prairies de Ray-grass avait été semée au printemps. L'étude a été limitée à la période comprise entre le 26 mai et le I7 juillet, les vaches ayant reçu une alimentation complémentaire (drèches et pulpes) dès les 20 juillet. En outre, nous avons éliminé la période du 2 I juin au 
I er juillet pendant laquelle en $\mathrm{I}$ j jours les vaches ont pâturé trois prairies différentes. Lorsque les vaches ont pâturé pour la première fois (Io au I 6 juin) la prairie de Raygrass semée au printemps, les quantités de lait et de matières grasses produites ont augmenté de façon spectaculaire (fig. $r$, tableau 4) pour diminuer ensuite quand les vaches ont pâturé la prairie suivante de dactyle. Inversement, le taux butyreux a diminué lorsque les vaches ont pâturé la prairie de ray-grass et il a augmenté lorsqu'elles ont pâturé la prairie de dactyle.

\section{TABLEAU 4}

Variations de la production laitière, du taux butyreux et de la production de matières grasses en fonction de la nature du paturage

(Jouy I958: valeurs moyennes observées sur I 2 vaches au pâturage)

\begin{tabular}{|c|c|c|c|c|c|c|c|c|c|}
\hline \multirow{2}{*}{$\begin{array}{c}\text { Nature } \\
\text { du pâturage }\end{array}$} & \multirow{2}{*}{$\begin{array}{l}\text { Date } \\
\text { d'exploi- } \\
\text { tation }\end{array}$} & \multirow{2}{*}{$\begin{array}{l}\text { Temps } \\
\text { de } \\
\text { séjour }\end{array}$} & \multirow{2}{*}{\begin{tabular}{|} 
Diminution \\
théorique \\
de la pro- \\
duction \\
laitière $(\mathrm{kg})$
\end{tabular}} & \multicolumn{3}{|c|}{ Variations moyennes (1) } & \multicolumn{3}{|c|}{ Variations immédiates $\left({ }^{2}\right)$} \\
\hline & & & & $\begin{array}{l}\text { Lait } \\
(\mathrm{kg})\end{array}$ & $\begin{array}{c}\text { TB } \\
(\text { g p p. } 1000)\end{array}$ & $\begin{array}{l}\mathrm{MG} \\
(g)\end{array}$ & $\begin{array}{l}\text { Lait } \\
(\mathrm{kg})\end{array}$ & $\begin{array}{c}\text { TB } \\
(\mathrm{g} \text { p. } 1000)\end{array}$ & $\begin{array}{l}\mathrm{MG} \\
(\mathrm{g})\end{array}$ \\
\hline $\begin{array}{l}\text { Dactyle } \ldots \ldots \ldots \ldots \ldots \\
\text { Ray-grass } \ldots \ldots \ldots \ldots \\
\text { Ray-grass } \\
\text { (semis } \\
\text { de }\end{array}$ & $\left|\begin{array}{rr}26 / 5 \text { au } & 4 / 6 \\
5 / 6 \text { au } & 9 / 6\end{array}\right|$ & $\begin{array}{r}10 \\
5\end{array}$ & $\begin{array}{l}-0,5 \\
-0,3\end{array}$ & $\begin{array}{l}-1,3 \\
+1,3\end{array}$ & $\begin{array}{l}-0,6 \\
-1,6\end{array}$ & -2 & $\begin{array}{l}\mid-0,8 \\
+0,2\end{array}$ & $\begin{array}{l}+1,3 \\
-1,3\end{array}$ & $\begin{array}{l}-10 \\
-47\end{array}$ \\
\hline 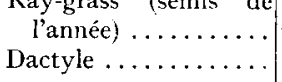 & $\mid \begin{array}{r}10 / 6 \text { au } 16 / 6 \\
7 / 6 \text { au } 21 / 6\end{array}$ & $\begin{array}{l}7 \\
5\end{array}$ & $-0,{ }^{\prime}$ & $-1,3^{\prime}$ & 0 & -2 & $-1,2$ & $+0,8$ & -29 \\
\hline $\begin{array}{l}\text { Dactyle .. } \\
\text { Ray-grass } \\
\text { Dactyle .. }\end{array}$ & $\begin{array}{r}1 / 7 \text { au } \quad 5 / 7 \\
6 / 7 \text { au } 12 / 7 \\
13 / 7 \text { au } 17 / 7\end{array}$ & $\begin{array}{l}5 \\
7 \\
5\end{array}$ & $\begin{array}{l}-0,3 \\
-0,3\end{array}$ & $\begin{array}{r}+0,1 \\
0,9\end{array}$ & $\begin{array}{l}-0,4 \\
\therefore 0,6\end{array}$ & - 19 & $\begin{array}{l}3+1,0 \\
0\end{array}$ & $\begin{array}{l}-0,8 \\
-0,7\end{array}$ & $\begin{array}{l}1 \\
-\quad 28\end{array}$ \\
\hline
\end{tabular}

(1) Variations moyennes : différences entre les valeurs moyennes observées au cours du séjour sur les deux prairies (par jour et par vache).

${ }_{\left({ }^{2}\right)}$ Variations immédiates : différences entre les valeurs movennes observées pendant les deux demiers jours sur une prairie et les deux premiers jours sur la prairie suivante (par jour et par vache).

Les quantités de lait et de matì̀res grasses ont de nouveau augmenté, mais beaucoup moins, lors de la deuxième exploitation ( 6 au I 2 juillet) de la prairie de Ray-grass semée au printemps, mais 1 a taux butyreux n'a pas diminué.

En dehors de ces deux périodes il y a eu peu de variations dues aux changements de prairies; les productions de lait et de matières grasses ont évolué normalement et le taux butyreux a lui-même paı varié.

\section{La Minière I95S}

Le lot expérimental de I9 vaches a pâturé des prairies de Ray-grass anglais et italien, de Fléole, de Fétuque des prés et de Dactyle associé ou non à de la Luzerne. I'été particulièrement pluvieux avait favorisé la croissance du Trèfle blanc, surtout dans les prairies de Ray-grass anglais et de Fléole très dégradées. Le temps de séjour 


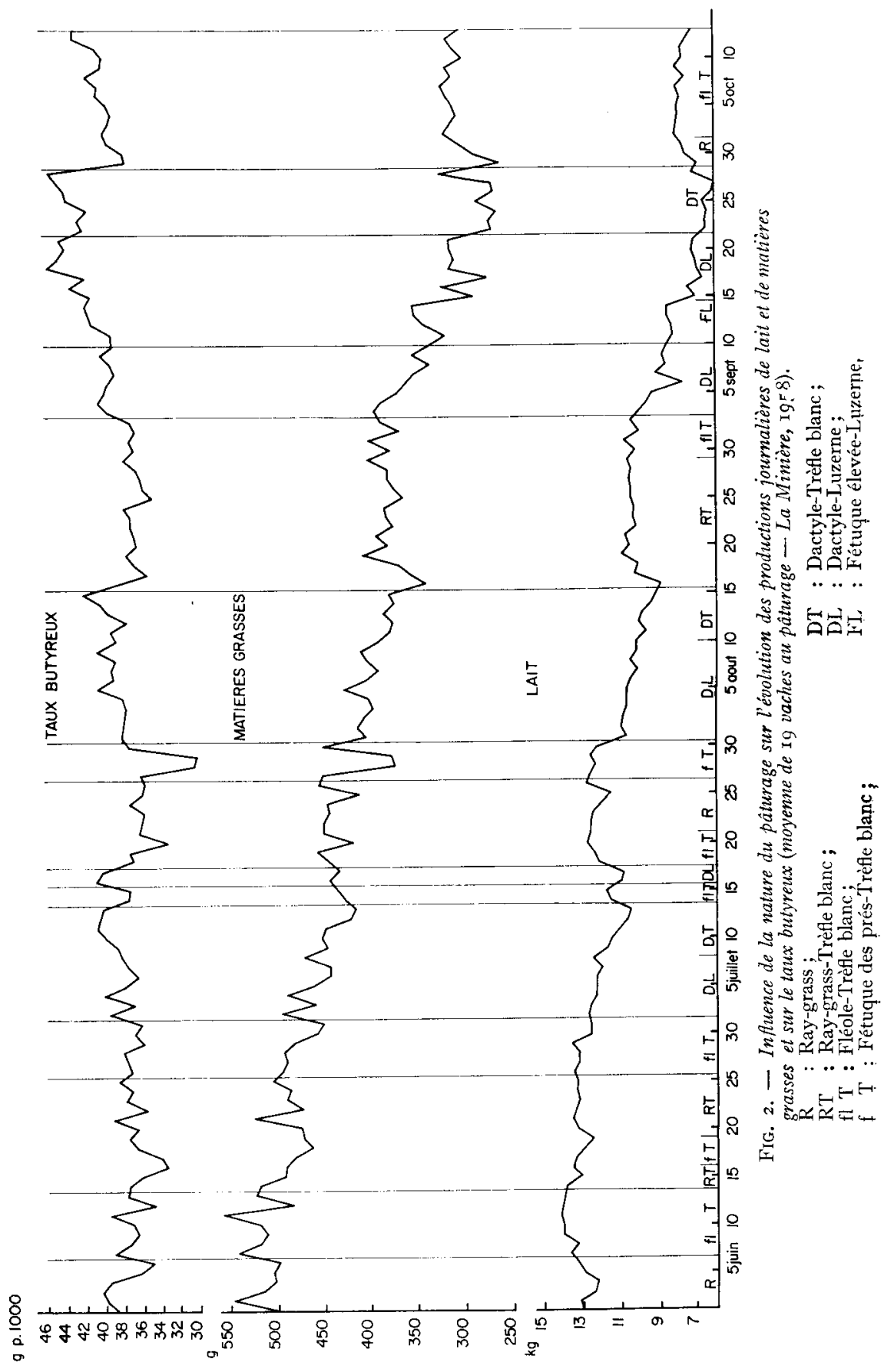


sur certaines prairies ayant été très court, nous avons groupé certaines prairies de variétés ou d'espèces différentes qui ont eu des actions identiques sur la quantité et la composition du lait. Les pâturages ont ainsi été groupés à partir du $\mathrm{I}^{\mathrm{er}}$ juin en I2 prairies ou groupes de prairies (fig. 2, tableau 5).

\section{TABLEAU 5}

Variations de la production laitière, du taux butyreux et de la production de matières grasses en fonction de la nature du pâturage

(La Minière 1958: valeurs moyennes observées sur 19 vaches au pâturage)

\begin{tabular}{|c|c|c|c|c|c|c|c|c|c|}
\hline \multirow{2}{*}{ Nature de pâturage } & \multirow{2}{*}{$\begin{array}{l}\text { Date } \\
\text { d'exploi- } \\
\text { tation }\end{array}$} & \multirow{2}{*}{$\begin{array}{l}\text { Temps } \\
\text { de } \\
\text { séjour }\end{array}$} & \multirow{2}{*}{$\mid \begin{array}{c}\text { Diminution } \\
\text { théorique } \\
\text { de la pro- } \\
\text { duction } \\
\text { laitière }(\mathrm{kg})\end{array}$} & \multicolumn{3}{|c|}{ Variations moyennes } & \multicolumn{3}{|c|}{ Variations immédiates } \\
\hline & & & & $\begin{array}{l}\text { Lait } \\
(\mathrm{kg})\end{array}$ & $\begin{array}{c}\text { TB } \\
(\mathrm{g} \text { p. } 1000)\end{array}$ & $\begin{array}{l}\mathrm{MG} \\
(\mathrm{g})\end{array}$ & $\begin{array}{c}\text { Lait } \\
(\mathrm{kg})\end{array}$ & $\begin{array}{c}\text { TB } \\
(\mathrm{g} \text { p. } 1000)\end{array}$ & $\begin{array}{l}\text { MG } \\
(\mathrm{g})\end{array}$ \\
\hline Ray-grass ......... & $1 / 6$ au $\quad 6 / 6$ & 6 & $\ldots 0,3$ & $+0,3$ & $-1,0$ & +3 & $+0,3$ & $+2,5$ & \\
\hline $\begin{array}{l}\text { Fléole-trèfle blanc .... } \\
\text { Ray-grass et fétuque }\end{array}$ & $7 / 6$ au $13 / 6$ & 7 & $\begin{array}{r}-0,3 \\
-0,5\end{array}$ & $\left\{\begin{array}{r}T \\
-0,0 \\
-0,6\end{array}\right.$ & $-0,8$ & -33 & $-0,6$ & $+0,5$ & +2 \\
\hline des prés-trèfle blanc. & 11 s/6 au $25 / 6$ & 12 & $-0,4$ & $-0,2$ & $+0,3$ & -11 & $-0,1$ & $-0,4$ & - 4 \\
\hline Dactyle-trèfle blanc- & $20 / 0201 / 1$ & 0 & $-0,4$ & $-1,3$ & $+1,8$ & -24 & 0 & $+1,5$ & +23 \\
\hline $\begin{array}{l}\text { dactyle luzerne } \ldots . . \\
\text { Ray-grass et fléole-trèfle }\end{array}$ & $2 / 7$ au $13 / 7$ & 12 & $-0,4$ & $\div 0,6$ & $-2,8$ & -12 & $+1,7$ & $-3,3$ & +31 \\
\hline $\begin{array}{r}\text { blanc } \ldots \ldots \ldots \ldots \\
\text { Fétuque des prés-trèfle }\end{array}$ & $18 / 7$ au $26 / 7$ & 9 & $-0,2$ & 0 & $-2,4$ & -29 & $+1,0$ & $-0,8$ & +2 \\
\hline $\begin{array}{r}\text { blanc } \ldots \ldots \ldots \ldots \\
\text { Dactyle luzerne et dac- }\end{array}$ & $27 / 7$ au $30 / 7$ & 4 & $-0,4$ & $-2,1$ & $+5,5$ & -17 & $-1,6$ & $+4,3$ & -63 \\
\hline $\begin{array}{l}\text { tyle-trèfle blanc .... } \\
\text { Ray-grass trèfle blanc. } \\
\text { Dactyle et fétuque éle- }\end{array}$ & $\mid \begin{array}{lr}31 / 7 \text { au } & 15 / 8 \\
16 / 8 \text { au } & 2 / 9\end{array}$ & $\begin{array}{l}16 \\
18\end{array}$ & $\begin{array}{l}-0,5 \\
-0,5\end{array}$ & $\begin{array}{l}-0,1 \\
-2,2\end{array}$ & $\begin{array}{r}-2,3 \\
-\quad 4,4\end{array}$ & $\begin{array}{rr}- & 18 \\
- & 45\end{array} \mid$ & $\begin{array}{l}+0,3 \\
-0,4\end{array}$ & $\begin{array}{l}-4,5 \\
+\quad 3,2\end{array}$ & $\begin{array}{r}-28 \\
+\quad 12\end{array}$ \\
\hline $\begin{array}{l}\text { vée-luzerne } \ldots \ldots \ldots \\
\text { Dactvle trèfle blanc .. }\end{array}$ & $\begin{array}{r}3 / 9 \text { au } 21 / 9 \\
0\end{array}$ & 19 & $-0,3$ & $-1,7$ & $+1,9$ & -54 & $-0,6$ & $-1,9$ & -43 \\
\hline Ray-grass et fléole-trèfle & 22/9 au $28 / 9$ & & $\longrightarrow 0,2$ & $+0,8$ & $-3,1$ & +28 & $+1,0$ & $-3,0$ & +13 \\
\hline blanc .............. & $29 / 9$ au $13 / 1$ & 15 & & & & & & & \\
\hline
\end{tabular}

\section{Production laitière.}

I a production laitière a subi de brusques variations lors des changements de pâturage. C'est ainsi que lors de leurs trois séjours sur des prairies à base de Dactyle, les vaches ont produit en moyenne pendant les deux premiers jours de pâturage sur ces prairies, respectivement 0, I, 6 et $0,4 \mathrm{~kg}$ de lait par vache de moins que pendant les deux jours précédents où elles avaient pâturé des prairies à base de Ray-grass, de Fléole et de Fétuque des prés. Inversement, durant les deux premiers jours de pâturage des prairies à base de Ray-grass les vaches ont produit respectivement $I, 7,0,3$ et $I, 0 \mathrm{~kg}$ de lait par vache de plus que durant les deux jours précédents où elles avaient pâturé des prairies à base de Dactyle.

La décroissance de production laitière a été également plus importante quand les vaches ont pâturé des prairies de Dactyle. Les équations des droites de régression liant la prođuction laitière journalière $y$ (exprimée en $\mathrm{kg}$ ) et le temps de séjour 
$x$ (exprimé en jours) sur les prairies à base de Ray-grass, Fétuque des prés ou Fléole d'une part (équation I) et sur les prairies à base de Dactyle d'autre part (équation 2), ont été en effet :

$$
\begin{array}{lll}
y=\mathrm{I}_{4}, \mathrm{I} 7-0,0475 x & \text { avec } r=-0,945 \quad \text { (équation } \mathrm{I}), \\
y=\mathrm{I}_{4}, 33-0,0626 x & \text { avec } r=-0,956 \quad \text { (équation 2). }
\end{array}
$$

Lorsque les vaches ont pâturé des prairies à base de Dactyle, la diminution de la production laitière due à l'avancement de la lactation a donc été de I,88 kg de lait par vache et par mois, soit I3,I p. Ioo alors qu'elle a été normale : I, $42 \mathrm{~kg}$ de lait par vache et par mois, soit Io p. Ioo, quand les vaches ont pâturé des prairies à base de Ray-grass, Fétuque des prés ou de Fléole.

Au total, compte tenu de l'avancement de la lactation, durant leurs trois séjours sur les prairies de Dactyle, les vaches ont en moyenne produit chaque jour respectivement $0,9, \mathbf{I}, 7$ et $I, 7 \mathrm{~kg}$ de lait par vache de moins que durant leurs trois séjours précédents sur les prairies à base de Ray-grass. Inversement, durant leurs séjours sur ces dernières prairies, les vaches ont en moyenne produit chaque jour respectivement, I,o, 0,6 et $\mathrm{I}, \mathrm{o} \mathrm{kg}$ de lait par vache de plus que durant leurs séjours précédents sur des prairies de Dactyle.

Il ne semble pas que la production laitière ait varié entre les prairies à base de Ray-grass, de Fétuque des prés ou de Fléole-Trèfle blanc; toutefois elle a été un peu plus élevée quand les vaches ont pâturé des prairies de Fléole -Trèfle blanc. Da même, la production laitière a été un peu plus faible lorsque les vaches ont pâturé des prairies de Dactyle que des prairies de Dactyle-Luzerne.

\section{Taux butyreux.}

Le taux butyreux a présenté des variations systématiquement inverses de celles de la quantité de lait.

Il a répondu brutalement aux changements de pâturage. C'est ainsi que lors des séjours des vaches sur les trois prairies de Dactyle, le taux butyreux moyen des deux premiers jours sur ces prairies a été respectivement supérieur de $1,5,4,3$ et 3,2 $\mathrm{g}$ p. I ooo à celui des deux jours précédents sur des prairies à base de Ray-grass, Fétuque des prés ou Fléole. Inversement, quand les vaches ont quitté les prairies à base de Dactyle pour pâturer les prairies à base de Ray-grass le taux butyreux moyen des deux premiers jours sur ces prairies a été respectivement inférieur de $3,3,4,5$ et 3,o g p. I 000 à celui des deux jours précédents sur les prairies de Dactyle.

Le taux butyreux moyen durant le séjour sur chaque prairie a varié dans le même sens. Il a été respectivement supérieur de $\mathrm{I}, 8,5,5$ et 4,4 $\mathrm{g}$ p. I ooo durant les trois séjours sur les prairies de Dactyle à celui durant les séjours précédents sur les prairies de Ray-grass, Fétuque des prés ou Fléole. Inversement, durant les trois séjours sur ces dernières prairies, il a été respectivement inférieur de $2,8,2,3$ et 3,I g p. I ooo à celui durant les trois précédents séjours sur les prairies de Dactyle. Toutes ces différences sont significatives.

Le taux butyreux n'a pas varié entre les prairies de Ray-grass, de Fétuque des prés ou de Fléole-Trèfle blanc, sauf quand les vaches ont pâturé du 27 au 30 juillet une prairie de Fétuque des prés où il a été significativement plus faible que sur la prairie précédente de Fléole, pâturée du I 8 au 26 juillet. De même il semble que le 
taux butyreux ait été plus élevé lorsque les vaches ont pâturé des prairies de Dactyle pur que lorsqu'elles ont pâturé des prairies de Dactyle-Luzerne.

Les variations du taux butyreux étant en relation étroite avec celles dela quantité de lait, nous avons calculé les coefficients de corrélation entre le taux butyreux et la quantité de lait durant le temps de séjour des vaches sur chaque prairie à base soit de Dactyle, soit de Ray-grass, puis sut ces deux types de prairies groupées deux à deux (tableau 6).

\section{TABLEAU 6}

Coefficient de corrélation entre le taux butyreux et la quantité de lait à l'intérieur de chaque groupe de prairies de même nature puis en groupant deux à deux des prairies de natures différentes

(La Minière 1958)

\begin{tabular}{|c|c|c|}
\hline Nature du pâturage & Date d'exploitation & $\begin{array}{l}\text { Coefficients } \\
\text { de corrélation }\end{array}$ \\
\hline 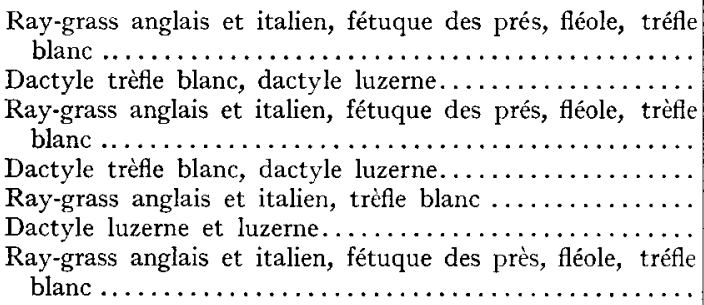 & $\begin{array}{r}1 / 6 \text { au } 1 / 7 \\
2 / 7 \text { au } 13 / 7 \\
18 / 7 \text { au } 30 / 7 \\
31 / 7 \text { au } 16 / 8 \\
17 / 8 \text { au } 2 / 9 \\
3 / 9 \text { au } 27 / 9 \\
28 / 9 \text { au } 13 / 10\end{array}$ & $\begin{array}{l}-0,196 \\
-0,646(1) \\
-0,275 \\
-0,499\left({ }^{(}\right) \\
+0,191 \\
-0,170 \\
-0,330\end{array}$ \\
\hline 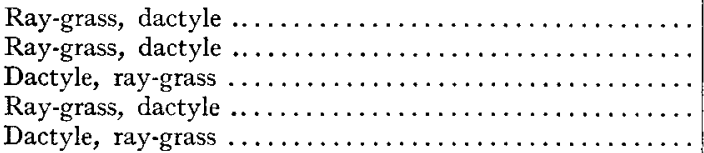 & 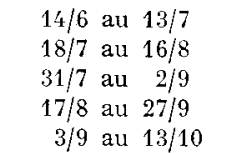 & $\begin{array}{r}-0,653\left({ }^{2}\right) \\
-0,753\left(^{2}\right) \\
-0,451\left(^{2}\right) \\
-0,893\left(^{2}\right) \\
-0,565\left(^{2}\right)\end{array}$ \\
\hline
\end{tabular}

(1) Significatif à la probabilité 0,05 .

(2) Significatif à la probabilité 0.01 .

On constate que :

- durant le séjour des vaches sur les prairies de Ray-grass, de Fétuque des prés ou de Fléole, les coefficients de corrélation ont été faibles et non significatifs. Il n'y a donc pas eu de liaison décelable entre taux butyreux et quantité de lait ;

- durant le séjour des vaches sur les prairies à base de Dactyle, les 3 coefficients de corrélation ont été négatifs, 2 l'étant significativement $(\mathrm{p}<0,05)$. Il a donc $\mathrm{pu}$ exister une liaison négative entre taux butyreux et quantité de lait, due vraisemblablement à la diminution accélérée de la production laitière quand les vaches ont pâturé ce type de prairie ;

- durant le séjour sur ces deux types de prairies groupées deux à deux, les 
coefficients de corrélation ont tous été négatifs et hautement significatifs ( $\mathrm{p}<\mathrm{o,0}$ ) Le taux butyreux et la production laitière ont donc évolué en sens inverse lors des changements de pâturage : Dactyle à Ray-grass ou Ray-grass à Dactyle.

\section{Production de matières grasses.}

La production journalière moyenne de matières grasses sur chaque prairie a diminué quand les vachas sont paszées d'une prairie à la suivante, sauf lors du dernier pâturage d'une prairie de Ray-grašs où elle a augmenté. Cependant elle a diminué plus rapidement quand les vaches sont passées d'une prairie de Ray-grass à une prairie de Dactyle que lors du passage inverse (fig. 2 ).

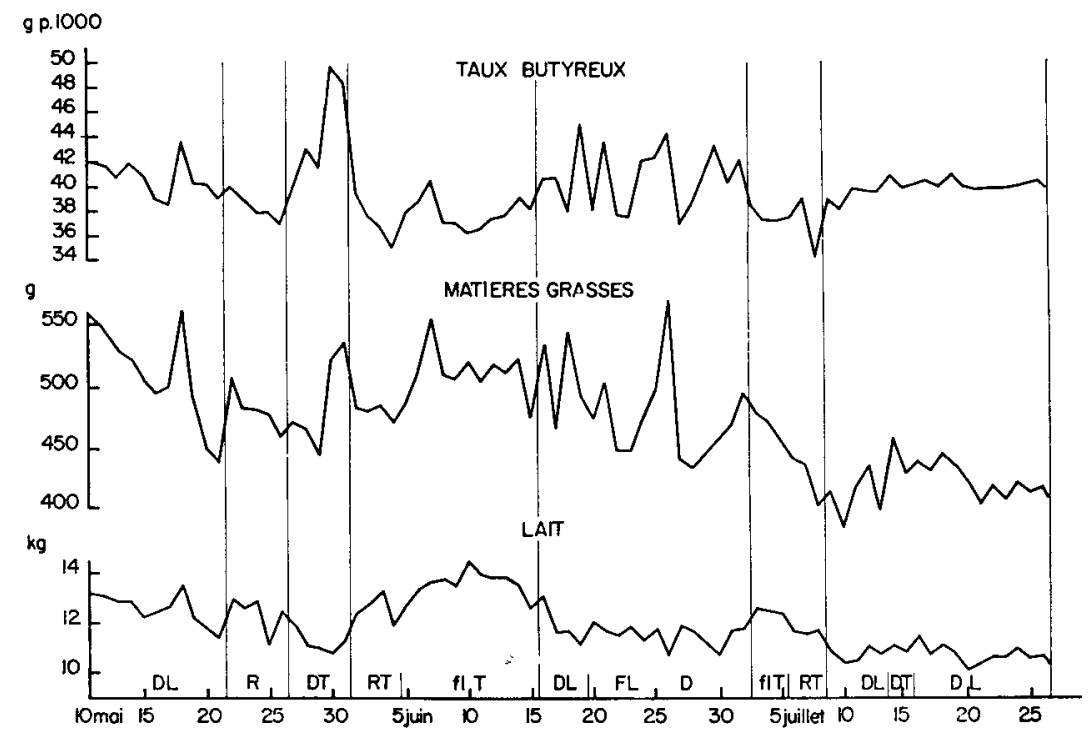

FIG. 3. - Influense do la nature du pâturage sur l'éyolution des productions journalières de lait et de matières grasses et sur le taux butyreux (moyenne de 18 vaches au paturage - La Minière, I959).

$\mathrm{R} \quad$ : Ray-grass ;

RT : Ray-grass-Trèfle blanc;

fl $T$ : Fléole-Trèfle blanc;

D : Dactyle;

DT : Dactyle-Trèfle blanc ;

DL : Dactyle-Luzerne;

FL : Fétuque élevée-Luzerne.

On peut donc dire que la quantité de matières grasses a évolué sensiblement comme la quantité de lait produite, ou que l'augmentation du taux butyreux quand les vaches ont pâturé les prairies de Dactyle a été insuffisante pour compenser la diminution de la production laitière.

\section{LA MINIÈRE I959}

L,e lot expérimental de 18 vaches a pâturé avec le troupeau les mêmes prairies que l'année précédente et une prairie de Fétuque élevée-Luzerne semée au printemps I958. L'année I959 a été caractérisée par un été extrêmement sec, et, par suite de la croissance très faible de l'herbe, les vaches ont reçı une alimentation complémen- 
taire (drèches) à partir du I ${ }^{\text {er }}$ août. Pour les mêmes raisons qu'en $195^{8}$, les pâturages du I I mai au 26 juillet ont été groupés en 7 prairies ou groupes de prairies, 4 à base de Dactyle et 3 à base de Ray-grass (fig. 3, tableau 7).

\section{Production laitière}

Comme en 1958 , la production laitière a subi de brusques variations lors des changements de pâturage. Lors des trois séjours sur les prairies à base de Dactyle, les vaches ont produit en moyenne durant les deux premiers jours de pâturage sur chacune de ces prairies, respectivement $0,3,0,7$ et I, I $\mathrm{kg}$ de lait par vache de moins que durant les deux jours précédents sur des prairies à base de Ray-grass. Inversement, elles ont produit lors de leurs deux premiers jours de séjour sur les prairies à base de Ray-grass, Fétuque des prés et Fléole $\mathrm{I}, 2, \mathrm{I}, 7$ et $0,8 \mathrm{~kg}$ de lait par vache de plus que lors des deux jours précédents sur des prairies à base de Dactyle.

TABLEAU 7

Variations de la production laiticire, du taux bulyreux et de la production de matières grasses en fonction de la nalure du pâturage.

(La Minière 1959: valeurs moyennes observées sur I 8 vaches au pâturage)

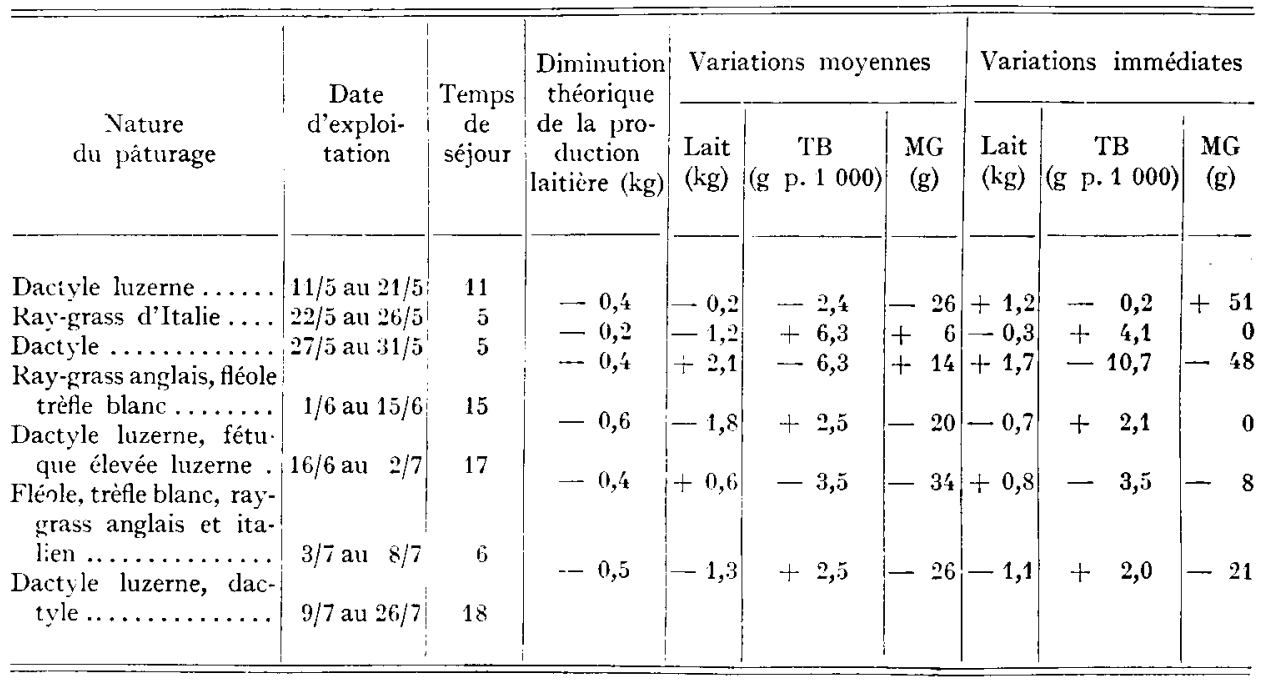

La décroissance de la production laitière a également été plus importante quand les vaches ont pâturé des prairies de Dactyle ou de Fétuque élevée. En effet, le coefficient de corrélation liant la production laitière journalière exprimée en $\mathrm{kg}$ et le temps de séjour (exprimé en jours) des vaches sur les différentes prairies est de - 0,75 ( $\mathrm{P}<0,0 \mathrm{I})$ sur les prairies de Dactyle et de Fétuqua élevée, et n'est que de - 0,23 (N.S.) sur les prairies de Ray-grass et de Fléole.

Au total, compte tenu de l'avancement de la lactation, durant leurs trois séjours sur des prairies de Dactyle, les vaches ont en moyenne produit chaque jour respectivement $I, O, \mathbf{I}, 2$ et $0,8 \mathrm{~kg}$ de lait par vache de moins que durant leurs trois séjours précédents sur des prairies à base de Ray-grass. Inversement, durant leurs séjours sur ces dernières prairies, les vaches ont en moyenne produit chaque jour respecti- 
vement $0,2,2,5$ et $\mathrm{r}, 0 \mathrm{~kg}$ de lait par vache de plus que durant leurs précédents séjours sur des prairies à base de Fétuque élevée ou de Dactyle.

La production laitière a cette année encore été un pau plus élevée quand les vaches ont pâturé des prairies à base de Fléole et de Trèfle blanc que des prairies à base de Ray-grass. Par contre, il y a un fait nouveau à signaler : la production laitière n'a pas varié entre les prairies à base de Iactyle et de Fétuque élevée.

\section{Taux butyreux}

I e taux butyreux a présenté des variations inverses de celles de la quantité de lait bien qu'elles aient été en partie masquées par des fluctuations journalières importantes.

Il a répondu brutalement aux changements de nature du pâturage. Lo taux butyreux moyen des deux premiers jours des trois séjours sur les prairies à base de Dactyle ou de Fétuque élevée a été supérieur respectivement de $4, \mathrm{I}, 2, \mathrm{I}$ et 2,0 $\mathrm{g}$ p. I ooo à celui des deux jours précédents sur des prairies à base de Ray-grass ou de Fléole-Trèfle blanc. Inversoment, quand les vaches ont quitté les prairies à base de Dactyle pour pâturer les prairies à base de Ray-grass ou de Fléole, le taux butyreux moyen des deux premiers jours sur ces prairies a été respectivement inférieur de 0,2, ro, 7 et $3,7 \mathrm{~g}$ p. I ooo à celui des deux jours précédents sur des prairies à base de Dactyle et de Fétuque élevée.

Le taux butyreux moyen au cours du séjour sur chaque prairie a varié de la même façon qu'en I958. Au cours des trois séjours des vaches sur les prairies de Dactyle et de Fétuque élevée, il a été respectivement supérieur de $6,3,2,5$ et $2,5 \mathrm{~g}$ p. I ooo à celui enregistré a cours des trois séjours sur des prairies de Ray-grass ou Fléole. Inversement, au cours des trois séjours sur ces dernières prairies il a été respectivement inférieur de $2,4,6,3$ et 3,5 p. I ooo à celui enregistré au cours des séjours précédents sur les prairies de Dactyle. Toutes ces variations ont été hautement significatives $(\mathrm{P}<\mathrm{O}, \mathrm{OI})$. Lorsque les vaches ont pâturé la prairie de Dactyle du 27 au $3 \mathrm{I}$ mai, le taux butyreux a augmenté très fortement $(6,3 \mathrm{~g} \mathrm{p}$. I ooo) pour diminuer très brutalement $(6,3 \mathrm{~g}$ p. I 000) quand les vaches ont pâturé la prairie suivante de Ray-grass. Ces variations semblent trop importantes pour être dues en totalité à la nature du pâturage ; elles ont peut-être été amplifiées par une autre cause.

Le taux butyreux n'a pas varié entre les prairies à base de Dactyle et de Fétuque élevée bien que les fluctuations du taux butyreux quand les vaches ont pâturé cette Fétuque élevée aient été très importantes.

\section{Production de matières grasses}

La nature du pâturage n'a pas eu d'influence nette sur la quantité journalière moyenne de matières grasses produite. Elle a diminué quand les vaches sont passées d'une prairie à la suivante, sauf sur les prairies de I)actyle et de Fléole pâturées respectivement $d u 27$ au 3 I mai et $d u I^{\text {er }}$ au ${ }_{5}$ juin. Les variations du taux butyreux en sens inverse de la production laitière ont dlonc été assez importantes pour "tamponner "les variations de la production de matières grasses. Ces résultats se trouvent confirmés par les variations au moment cles changements de prairies. En effet, lors des trois séjours sur des prairies de Dactyle faisant suite à des prairies à base de Ray-grass ou de Fléole-'Trèfle blanc, la production moyenne des deux premiers jours a augmenté dans un cas et diminué dans les deux autres; de même, lors des 
trois séjours sur des prairies de Ray-grass ou de Fléole-'Trèfle blanc faisant suite à des prairies de Dactyle, la production moyenne des deux premiers jours est restée invariable dans deux cas et a diminué dans le troisième.

\section{JouY 1959}

Enn I959, à Jouy, nous avons apporté de l'herbe coupée à un lot de vaches maintenues en stabulation afin de voir si la nature de l'herbe modifiait la production laitière et le taux butyreux dans les mêmes proportions qu'au pâturage.

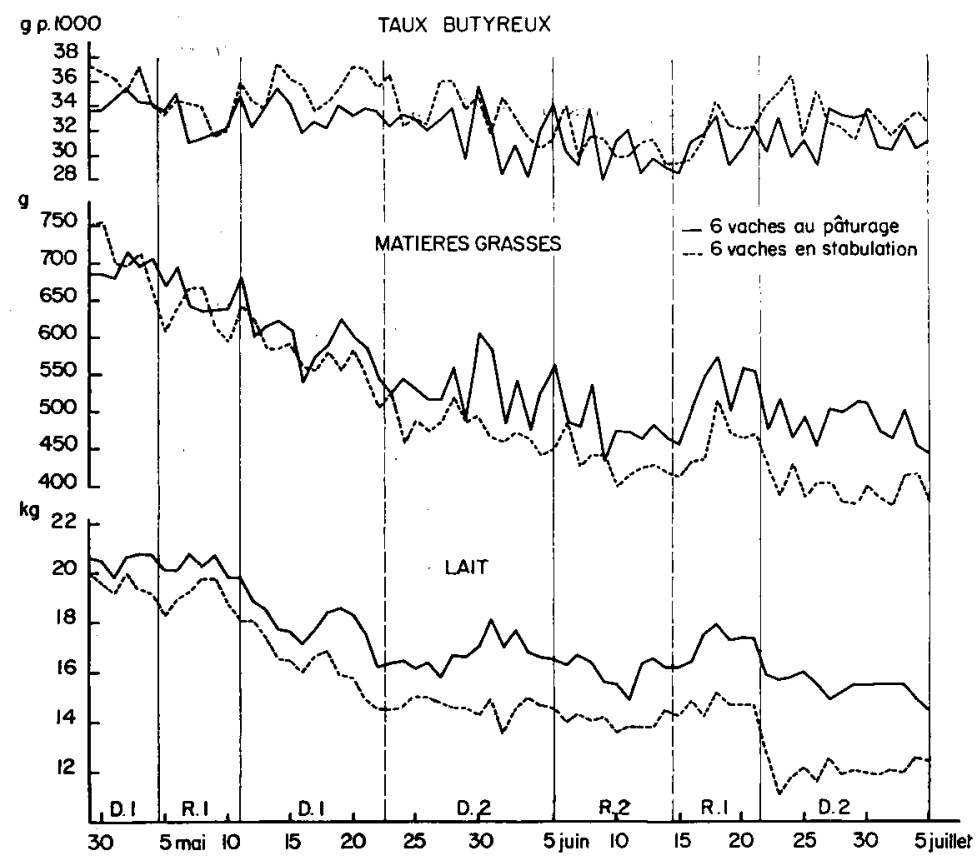

FIts. 4. - Influence de la nature du paturage sur l'évolution des productions journalières de lait et de matières grasses et sur le taux butyreux (myenne de 6 vaches au páturage et de 6 vaches en stabulation $\rightarrow$ Jouy, 1959). $\mathrm{R}_{\mathrm{I}}$ : Ray-grass en première exploitation; $\quad \mathrm{Dr}_{\mathrm{r}}$ : Dactyle en première exploitation;

$\mathrm{R}_{2}$ : Ray-grass en deuxième exploitation; $\quad \mathrm{D}_{2}$ : Dactyle en deuxième exploitation.

Pour cela, nous avons constitué deux lots semblables de 6 vaches chacun : un lot est sorti au pâturage, l'autre demeuré en stabulation a reçu de l'herbe fauchée chaque matin dans une bande réservée à la fauche dans les prairies pâturées par le premier. Pour se rapprocher des conditions du pâturage, les vaches en stabulation avaient accès deux fois par jour de I I h à $\mathrm{I} 4 \mathrm{~h}$ et de $20 \mathrm{~h}$ à $5 \mathrm{~h}$ à une aire de stabulation extérieure. Les quantités individuelles de matière verte et de matière sèche d'herbe consommées ont été enregistrées suivant les méthodes précédemment décrites.

Par suite de la sécheresse estivale, les vaches des deux lots ont reçu des fourrages verts d'origine variée (avoine - vesce - luzerne) en complément à partir du I 6 juillet. La période de comparaison retenue va donc du 29 avril au 5 juillet (fig. 4 , tableaux 8 et 9). Durant cette période les vachas ont consommá du Dactyle ou du Ray-grass presque purs en première ou deuxième exploitation. 


\section{Production laitière}

la production laitière a présenté les mêmes variations dans les deux groupes aussi bien en valeurs moyennes (entre les prairies) qu'en valeurs journalières (à l'intérieur d'une même prairie). La production des vaches au pâturage a été cependant plus élevée que celle des vaches alimentées en stabulation.

\section{TABLEAU 8}

Variations de la production laitière, du taux butyreux et de la production de matieres grasses en fonction de la nature du pâturage

(Jouy 1959: valeurs moyennes observées sur 6 vaches au pâturage)

\begin{tabular}{|c|c|c|c|c|c|c|c|c|c|}
\hline \multirow{2}{*}{$\begin{array}{c}\text { Nature } \\
\text { du pâturage }\end{array}$} & \multirow{2}{*}{$\begin{array}{c}\text { Date } \\
\text { d'exploi- } \\
\text { tation }\end{array}$} & \multirow{2}{*}{$\begin{array}{c}\text { Temps } \\
\text { de } \\
\text { séjour }\end{array}$} & \multirow{2}{*}{$\begin{array}{c}\text { Diminution } \\
\text { théorique } \\
\text { de la pro- } \\
\text { duction } \\
\text { laitière }(\mathrm{kg})\end{array}$} & \multicolumn{3}{|c|}{ Variations moyennes } & \multicolumn{3}{|c|}{ Variations immédiates } \\
\hline & & & & $\begin{array}{l}\text { Lait } \\
(\mathrm{kg})\end{array}$ & $\begin{array}{c}\mathrm{TB} \\
(\mathrm{g} p .1000)\end{array}$ & $\begin{array}{c}\mathrm{MG} \\
(\mathrm{g})\end{array}$ & $\begin{array}{l}\text { Lait } \\
(\mathrm{kg})\end{array}$ & 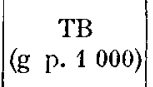 & $\begin{array}{c}\mathrm{MG} \\
(g)\end{array}$ \\
\hline 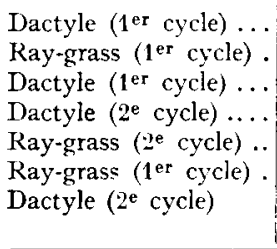 & $\mid \begin{array}{rr}29 / 4 \text { au } & 1 / 5 \\
5 / 5 \text { au } & 11 / 5 \\
11 / 5 \text { au } & 22 / 5 \\
23 / 5 \text { au } & 5 / 6 \\
5 / 6 \text { au } & 14 / 6 \\
15 / 6 \text { au } & 21 / 6 \\
22 / 6 \text { au } & 5 / 7\end{array}$ & $\begin{array}{r}6 \\
7 \\
12 \\
14 \\
10 \\
7 \\
14\end{array}$ & $\begin{array}{l}-0,5 \\
-0,7 \\
-0,8 \\
-0,7 \\
-0,4 \\
-0,6\end{array}$ & $\begin{array}{r}-0,3 \\
-2,2 \\
-1,3 \\
-0,6 \\
+1,1 \\
-1,8\end{array}$ & $\begin{array}{l}-1,5 \\
+\quad 0,7 \\
-1,4 \\
-1,5 \\
+\quad 0,3 \\
+\quad 0,6\end{array}$ & $\left|\begin{array}{ll}- & 39 \\
- & 57 \\
- & 66 \\
- & 46 \\
+ & 42 \\
- & 44\end{array}\right|$ & $\left|\begin{array}{r}-0,3 \\
-1,6 \\
-0,5 \\
-0,2 \\
-0,1 \\
-1,6\end{array}\right|$ & $\begin{array}{ll} & 0 \\
+ & 1,0 \\
- & 0,8 \\
- & 0,3 \\
+ & 0,5 \\
+ & 0.3\end{array}$ & $\begin{array}{r}-21 \\
-\quad 29 \\
-\quad 27 \\
-\quad 15 \\
+\quad 6 \\
-\quad 72\end{array}$ \\
\hline
\end{tabular}

TABLEAU 9

Variations de la production laitière, du taux butyreux et de la production de matières grasses en fonction de la nature du pâturage

(Jouy I959: valeurs moyennes observées sur 6 vaches en stabulation)

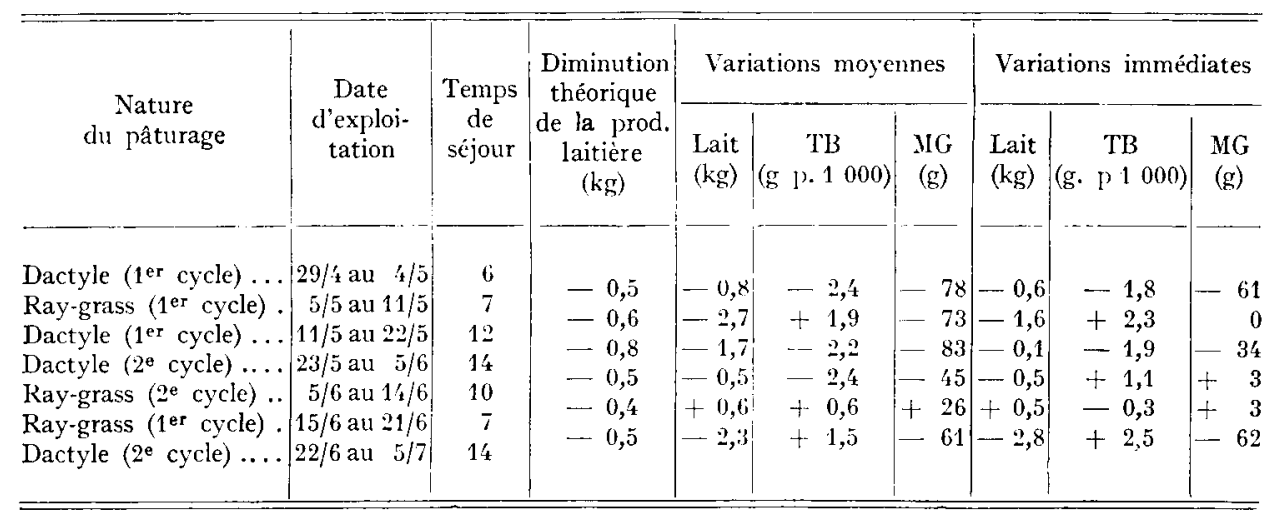


Les vaches ont produit en moyenne durant les deux premiers jours sur les deux prairies de Dactyle respectivement I,6 et I,6 kg de lait par vache au pâturage et I, 6 et $2,8 \mathrm{~kg}$ de lait par vache en stabulation de moins que pendant les deux jours précédents sur des prairies de Ray-grass. Par contre, la production laitière n'a guère varié lors du changement inverse quand les vaches sont passées des prairies de Dactyle aux prairies de Ray-grass.

De même, compte tenu de l'avancement de la lactation durant leurs deux séjours sur les prairies de Dactyle, les vaches ont en moyenne produit chaque jour respectivement I, 5 et I, $2 \mathrm{~kg}$ de lait par vache au pâturage et $2, \mathrm{I}$ et I, $8 \mathrm{~kg}$ de lait par vache en stabulation de moins que durant leurs séjours sur les prairies prédécentes de Ray-grass, mais la production moyenne n'a guère varié lors du changement inverse. La production laitière journalière s'est donc simplement' arrêtée de décroître pour demeurér sensiblement constante durant le séjour des vaches sur les prairies de Ray-grass. Il en a été de même quand les vaches sont passées d'une prairie de Dactyle en première exploitation à une autre en deuxième exploitation.

\section{Taux butyreux}

Le taux butyreux a évolué d'une façon presque semblable dans les deux lots malgré les fluctuations journalières importantes dues au petit nombre de vaches par lot.

Comme dans les observations des années précédentes, le taux butyreux moyen a été plus élevé quand les vaches ont consommé du Dactyle que lorsqu'elles ont consommé du Ray-grass, mais les variations ont été faibles, surtout chez le groupe au pâturage où elles sont demeurées comprises entre 0,3 et $1,5 \mathrm{~g} \mathrm{p}$. I ooo.

Il faut noter enfin une baisse relativement importante du taux butyreux (en moyenne respectivement 2,2 et $I, 4 \mathrm{~g}$ p. I ooo en stabulation et au pâturage) quand les vaches sont passées d'une prairie de Dactyle en première exploitation à une autre en deuxième exploitation.

\section{Production de matières grasses}

La production de matières grasses a évolué de façon semblable dans les deux groupes. Les variations du taux butyreux ayant été relativement faibles, elle a présenté les mêmes variations que la production laitière. Elle a donc diminué rapidement pendant le séjour des vaches sur les prairies de Dactyle ; elle s'est arrêtée de décroître et est demeurée sensiblement constante durant le séjour sur les prairies de Ray-grass.

\section{Quantité consommée}

Les vaches alimentées en stabulation ont consommé systématiquement plus de Ray-grass que de Dactyle, en matière sèche :

9,6 kg pour le Dactyle en fin de première exploitation.

I I, I kg pour le Dactyle en deuxième exploitation.

$\mathrm{I} 2,4 \mathrm{~kg}$ pour le Ray-grass en première et deuxième exploitation. 


\section{Jouy 1960}

Comme en I959, à Jouy, nous avons etu deux lots semblables mais de 8 vaches chacun, l'un au pâturage, l'autre en stabulation. En outre, la digestibilité de l'herbe des différentes prairies a été mesurée sur un lot de 5 béliers de façon à pouvoir estimer les quantités d'éléments nutritifs consommés par les vaches.

La période de transition à la mise à l'herbe terminée, les vaches n'ont consommé

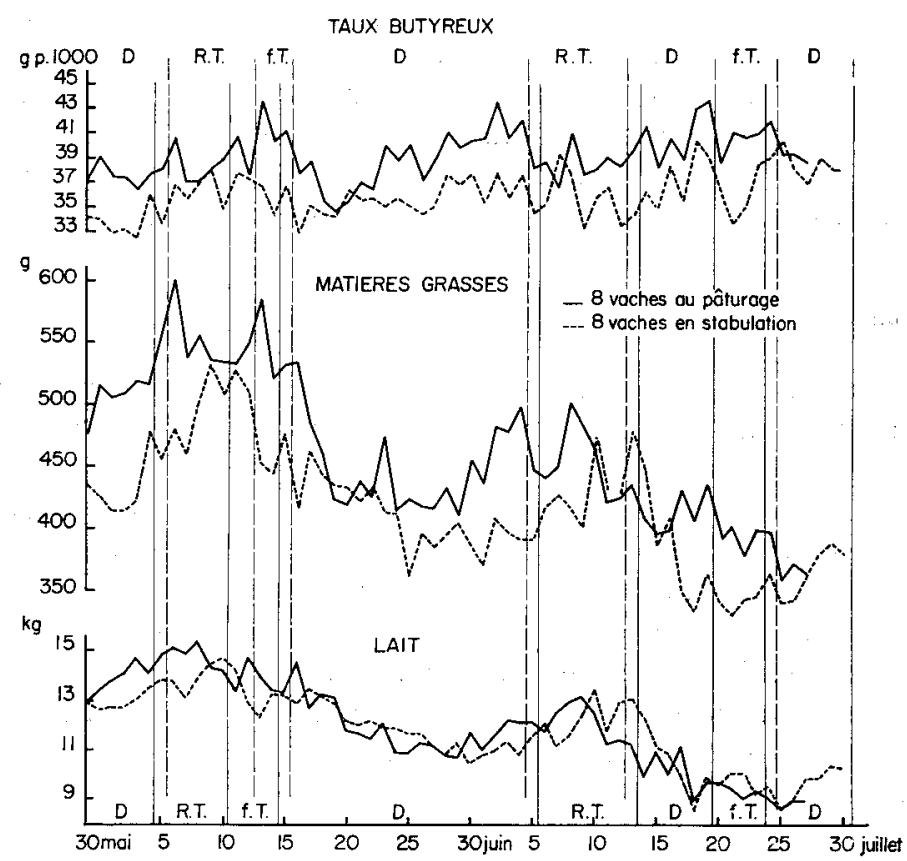

FIG. 5. - Influence de la nature du pâturage sur l'évolution des productions journalières de lait et de matières grasses et sur le taux butyreux (moyenne de 8 vaches au paturage et de 8 vaches en stabulation - Jouy, ig6o). RT : Ray-grass-Trèfle blanc exploité l'année du semis ;

f T : Fétuque des prés-Trèfle blanc exploité l'année du semis ;

D : Dactyle.

que du Dactyle jusqu'au 5 juin et elles ont reçu des drèches en complément del'herbe à partir du $I^{\text {er }}$ août. La période de comparaison est donc comprise entre le 30 mai et le 30 juillet (fig. 5, tableaux Io et II). Durant cette période, les vaches ont consommé du Ray-grass hybride et de la Fétuque des prés provenant de prairies semées au printemps et du Dactyle. Pour des raisons de commodité pratique, les changements de prairies n'ont pas toujours eu lieu exactement à la même date pour les deux groupes; il y a eu parfois un décalage de I ou 2 jours.

\section{Production laitière}

Ia production laitière a évolué d'une façon très semblable dans les deux groupes.

A l'inverse des années précédentes, elle a, très peu varié au moment des changements de prairies. Par contre, les productions moyennes durant le séjour sur chaque 


\section{TABIEAU IO}

Variations de la production laitière, du taux butyreux et de la production de matières grasses en fonction de la nature du pâturage

(Jouy 1960: valeurs moyennes observées sur 8 vaches au pâturage)

\begin{tabular}{|c|c|c|c|c|c|c|c|c|c|}
\hline \multirow{2}{*}{$\begin{array}{l}\text { Nature } \\
\text { du Fâturage }\end{array}$} & \multirow{2}{*}{$\begin{array}{l}\text { Date } \\
\text { d'exploi- } \\
\text { tation }\end{array}$} & \multirow{2}{*}{$\begin{array}{l}\text { Temps } \\
\text { de } \\
\text { séjour }\end{array}$} & \multirow{2}{*}{$\begin{array}{c}\text { Diminution } \\
\text { théorique } \\
\text { de la pro- } \\
\text { duction } \\
\text { taitiere (kg) }\end{array}$} & \multicolumn{3}{|c|}{ Variations moyennes } & \multicolumn{3}{|c|}{ Variations immédiates } \\
\hline & & & & $\begin{array}{l}\text { Lait } \\
(\mathrm{kg})\end{array}$ & $\begin{array}{c}\text { TB } \\
(\Leftrightarrow \text { p. } 1000)\end{array}$ & $\begin{array}{c}\mathrm{MG} \\
(\mathrm{g})\end{array}$ & $\begin{array}{l}\text { Lait } \\
(\mathrm{kg})\end{array}$ & $\begin{array}{c}\text { TB } \\
\text { (g p. } 1000)\end{array}$ & $\begin{array}{l}M G \\
(\mathrm{~g})\end{array}$ \\
\hline acty & $30 / 5$ an $4 / 6$ & 6 & $-0,3$ & $+1,0$ & +0.8 & + & 0,5 & + & t \\
\hline $\begin{array}{l}\text { He blane } \\
\text { 'amnée) ... }\end{array}$ & & 6 & & & & & & & \\
\hline $\begin{array}{l}\text { Fétuque-t } \\
\text { mis de }\end{array}$ & $i_{11 / 6: 11}$ & & & & & & & & \\
\hline $\begin{array}{l}\text { Dactyle .. } \\
\text { Ray-grass- }\end{array}$ & $\begin{array}{ll}15 / t i \text { aul } & 5 / 7\end{array}$ & 21 & $\begin{array}{cc}-0,6 \\
-\ldots & 0,6\end{array}$ & $\begin{array}{r}-2,0 \\
+0,3\end{array}$ & $\begin{array}{l}-1,6 \\
-0,4\end{array}$ & $\begin{array}{r}-95 \\
0\end{array}$ & $\left|\begin{array}{c}0,2 \\
0\end{array}\right|$ & $\begin{array}{l}-2,5 \\
-2,5\end{array}$ & $\begin{array}{l}-21 \\
-27\end{array}$ \\
\hline I $(\mathrm{sem}$ & $6 / 7$ au $13 / 7$ & 9 & $\cdots$ & -2 & + & -40 & $-0,9 \mid$ & + & -2 \\
\hline (se. & $14 / 7 \mathrm{a}$ & 6 & & $-0,8$ & 1 & -19 & $+0,2$ & 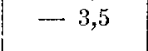 & $-2 t$ \\
\hline $\begin{array}{l}\text { mis de } \\
\text { Dactyle }\end{array}$ & $\begin{array}{l}20 / 7 \mathrm{a} \\
24 / 7 \mathrm{a}\end{array}$ & $\begin{array}{l}4 \\
4\end{array}$ & $--0,1$ & $-0,5$ & $-0,5$ & -20 & $-0,4$ & $-0,1$ & -10 \\
\hline
\end{tabular}

TABIEAU II

Variations de la production laiticre, du taux butlreux et de la production de matières grasses en fontion de la nature du pâturage.

(Jouy 1960: Valeurs moyennes observées sur 8 vaches en stabulation)

\begin{tabular}{|c|c|c|c|c|c|c|c|c|c|}
\hline \multirow{2}{*}{$\begin{array}{c}\text { Nature } \\
\text { du paturage }\end{array}$} & \multirow{2}{*}{$\begin{array}{l}\text { Date } \\
\text { d'exploi- } \\
\text { tation }\end{array}$} & \multirow{2}{*}{$\begin{array}{l}\text { Temps } \\
\text { de } \\
\text { séjour }\end{array}$} & \multirow{2}{*}{$\begin{array}{l}\text { Diminution } \\
\text { theorique } \\
\text { cle la pro- } \\
\text { duction } \\
\text { laitiore }(\mathrm{kg})\end{array}$} & \multicolumn{3}{|c|}{ Variations moyennes } & \multicolumn{3}{|c|}{ Variations immédiates } \\
\hline & & & & $\begin{array}{l}\text { Lait } \\
(\mathrm{kg})\end{array}$ & $\begin{array}{c}\mathrm{TB} \\
(\mathrm{g} \quad \mathrm{j}) .1000)\end{array}$ & $\begin{array}{l}\mathrm{MG} \\
(\mathrm{g})\end{array}$ & $\begin{array}{l}\text { Lait } \\
\text { (kg) }\end{array}$ & TB & $\begin{array}{l}\mathrm{MG} \\
(\mathrm{g})\end{array}$ \\
\hline Dactyle & $30 / 5$ au $5 / 6$ & 7 & & & & & & & \\
\hline $\begin{array}{l}\text { Ray-grass trifle blanc } \\
\text { (semis de l'annce) .. }\end{array}$ & $6 / 6$ at1 $12 / 6$ & 7 & $-0,2$ & $-2,1$ & $-0,8$ & $\begin{array}{l}+71 \\
-\quad 48\end{array}$ & $-1,2$ & $-2,1$ & $\begin{array}{r}+12 \\
-\quad 72\end{array}$ \\
\hline $\begin{array}{l}\text { mis de l'année) } \ldots . \\
\text { Dactyle ............ } \\
\text { Ray-grass trète blanc }\end{array}$ & $\mid \begin{array}{ll}13 / 6 \text { an } & 15 / 6 \\
16 / 6 \text { and } & 6 / 7\end{array}$ & $\begin{array}{r}3 \\
19\end{array}$ & $\begin{array}{l}-0, f_{1} \\
-0.6\end{array}$ & $\begin{array}{l}-1,1 \\
+\quad 0,3\end{array}$ & $\begin{array}{r}-0,4 \\
+0,2\end{array}$ & $\begin{array}{l}-50 \\
+\quad 16\end{array}$ & $\begin{array}{c}0 \\
+0,7\end{array}$ & $\begin{array}{l}-1,7 \\
-1,9\end{array}$ & $\begin{array}{l}-21 \\
+\quad 11\end{array}$ \\
\hline $\begin{array}{l}\text { (semis de l'année) .. } \\
\text { Dactyle ............. } \\
\text { Fétuque trèfle blanc (se. }\end{array}$ & $\mid \begin{array}{r}5 / 7 \text { an } 12 / 7 \\
13 / 7 \text { an } 19 / 7\end{array}$ & $\begin{array}{l}5 \\
7\end{array}$ & $\begin{array}{l}-0.3 \\
-0.2\end{array}$ & $\begin{array}{l}-1,2 \\
-1,1\end{array}$ & $\begin{array}{r}+\quad 1,3 \\
+\quad 0,5\end{array}$ & $\left\{\begin{array}{l}-28 \\
-51\end{array}\right.$ & $\begin{array}{r}+0,3 \\
+0,6\end{array}$ & $\begin{array}{l}+1,4 \\
-4,9\end{array}$ & $\begin{array}{l}+33 \\
-13\end{array}$ \\
\hline $\begin{array}{c}\text { mis de l'année) } \ldots . \\
\text { Dactyle } . . . . . . .\end{array}$ & $\begin{array}{l}20 / 7 \text { a.u } 24 / 7 \\
25 / 7 \text { au } 30 / 7\end{array}$ & $\begin{array}{l}5 \\
6\end{array}$ & --0.2 & 0 & $+1,9$ & +19 & $-0,6$ & $+0,4$ & -13 \\
\hline
\end{tabular}


prairie ont varié comme dans les observations précédentes. Compte tenu de l'avancement de la lactation, durant les deux séjours sur des prairies de Ray-grass, les vaches ont en moyenne produit chaque jour respectivement $\mathrm{I}, 3$ et $0,9 \mathrm{~kg}$ de lait par vache à la fois au pâturage et en stabulation de plus que durant les deux séjours précédents sur des prairies de Dactyle. Inversement, durant un séjour sur une prairie de Dactyle, les vaches ont en moyenne produit chaque jour $1,7 \mathrm{~kg}$ de lait par vache au pâturage et $0,9 \mathrm{~kg}$ de lait par vache en stabulation de moins que durant le séjour précédent sur une prairie de Ray-grass.

Quand les vaches, après avoir consommé soit du Ray-grass, soit du Dactyle, ont consommé de la Fétuque des prés, la production laitière a diminué. La diminution a surtout été importante chez le groupe alimenté en stabulation. Ce fait nouveau est à rapprocher de l'envahissement de cette jeune prairie de fétuque par les mauvaises herbes. Les vaches, surtout celles recevant l'herbe coupée, n'ont probablement pas pu consommer cette fétuque à volonté malgré le tri important qu'elles ont effectué dans la ration qui leur fut offerte.

\section{Taux butyreux.}

I,e taux butyreux a évolué d'une façon presque semblable dans les deux lots, bien qu'il ait été constamment plus faible dans le lot en stabulation.

Les vaches quittant la première prairie de Dactyle, le taux butyreux a augmenté brutalement et s'est maintenu élevé lors de la première exploitation des semis de l'année de Ray-grass et de Fétuque. Il a ensuite diminué temporairement quand les vaches ont quitté ces semis de l'année pour une prairie de Dactyle. C'est la première fois que nous enregistrons une augmentation du taux butyreux chez les vaches consommant du Ray-grass ou de la Fétuque des prés. Ensuite nous retrouvons des variations identiques à celles enregistrées les années précédentes ; lorsque les vaches ont consommé du Dactyle, le taux butyreux a été plus élevé que lorsqu'elles ont consommé du Ray-grass et de la Fétuque des prés en deuxième exploitation.

\section{Production de matières grasses}

La production de matières grasses a évolué comme la production laitière. Elle a augmenté rapidement et est demeurée élevée quand les vaches ont consommé l'herbe des jeunes prairies de l'année, surtout lors de la première exploitation du semis de Ray-grass puisque la production laitière et le taux butyreux ont augmenté. La production de matières grasses a diminué très rapidement entre le $I_{5}$ et le 20 juin quand les vaches sont revenues sur des prairies de Dactyle. Elle a ensuite augmenté en fin d'exploitation des prairies de Dactyle et surtout lors de la deuxième exploitation de la jeune prairie de Ray-grass, pour diminuer de nouveau brutalement, surtout chez le lot en stabulation, quand les vaches sont revenues du I3 au I9 juillet sur une prairie de Dactyle.

\section{Quantités d'éléments nutritifs consommées.}

Les vaches alimentées en stabulation ont consommé plus de Ray-grass que de Dactyle et surtout que de Fétuque (tableau I4). Rappelons à ce sujet que la jeune prairie de Fétuque était envahie de nombreuses mauvaises herbes autres quedes graminées. En général, la quantité consommée a varié brutalement lors des changements de prairies (fig. 6). 
La digestibilité du Ray-grass a été supérieure à celle du Dactyle et de la Fétuque des prés qui a eu une digestibilité anormalement basse à la deuxième exploitation (tableau I4). En moyenne le Ray-grass a eu un coefficient de digestibilité de la matière organique supérieur de 6,2 points à celui du Dactyle, et une valeur énergétique plus élevée : $0,78 \mathrm{UF} / \mathrm{kg}$ M.S. contre 0,70 pour la Fétuque et 0,72 pour le Dactyle.

Au total, avec le Ray-grass, les vaches ont consommé en moyenne chaque jour l'équivalent de 0,65 UF et $\mathbf{1}, 25$ UF par vache de plus respectivement qu'avec le Dactyle et la Fétuque des prés.

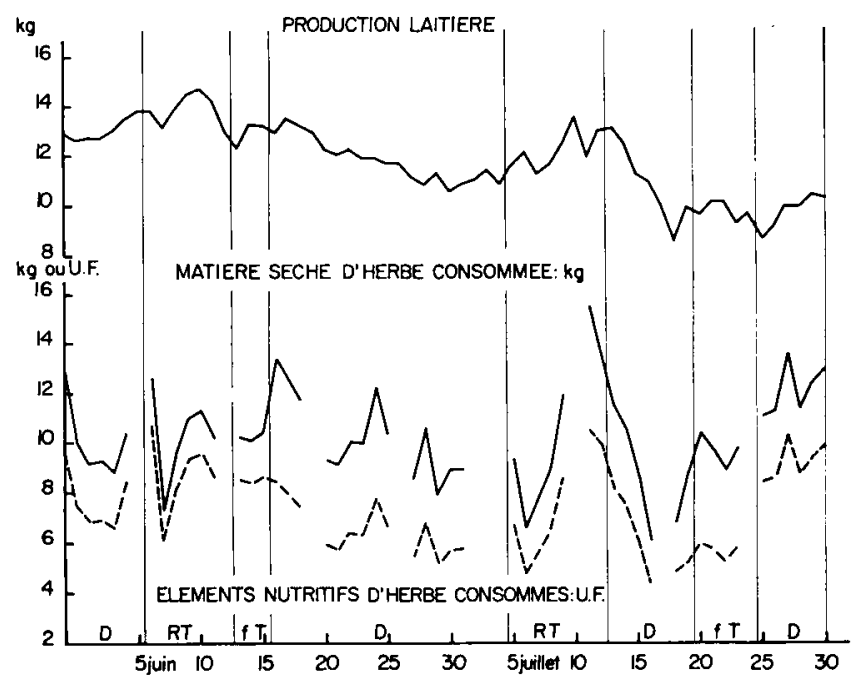

FIG. 6. - Influence de la nature du pâturage sur l'évolution de la production laitière journalière et surles quantités journalières de matière sèche et d'éléments nutritifs d'herbe consommées (moyenne de 8 vaches en stabulation - Jouy, 1960).

RT : Ray-grass-Trèfle blanc exploité l'année du semis;
f $\mathrm{T}:$ Fétuque des prés-Trèfle blanc exploité l'année du semis;
$\mathrm{D} \quad:$ Dactyle.

\section{L.A Minière ig6I}

Comme en I959 et I96o à Jouy, nous avons eu deux lots de vaches (Io vaches par lot), l'un au pâturage, l'autre en stabulation. L,es quantités de matière sèche d'herbe consommées par les vaches alimentées en stabulation ont été individuellement enregistrées et la digestibilité de 1'herbe de chaque prairie a été mesurée, à chaque exploitation, sur un lot de 5 béliers. En outre, lors des exploitations successives de certaines prairies, nous avons prélevé du jus de rumen par tubage, sur un certain nombre de vaches au pâturage et en stabulation. Ces prélèvements ont été effectués toujours à la même heure, au moment de la traite du soir et alors que les vaches consommaient l'herbe de la prairie depuis trois jours au moins. Les échantillons prélevés ont été analysés suivant les méthodes précédemment décrites pour déterminer leurs teneurs en acides acétique, propionique et butyrique.

Les prairies étudiées étaient à base de Ray-grass hybride, de Fétuque des prés 
et Fléole, et de Dactyle associé à du Trè̀le blanc, de Dactyle-Luzerne et de Luzerne pure (tableau 2). Par suite de la sécheresse et d'une superficie disponible insuffisante, les mesures d'alimentation des vaches en stabulation ont été arrêtées le ${ }^{\text {er }}$ septembre. La période de comparaison retenue va donc du I5 $_{5}$ mai au I $^{\text {er }}$ septembre pour le lot alimenté en stabulation et du I5 mai au 22 octobre pour le lot au pâturage (fig. 7 , tableaux I2 et I3).

Deux vaches, l'une au pâturage, l'autre en stabulation, ont été accidentées lors de l'exploitation, du r9 au 28 Juin, d'une prairie de Fétuque des prés-Fléole. Sur cette prairie, ainsi que sur les deux qui l'encadrent, les valeurs moyennes pour le lait, le taux butyreux et les matières grasses ont été calculées pour 9 vaches.

TABLEAU I2

Variations de la production laitière, du taux butyreux et de la production de matières grasses en fonction de la nature du pâturage

(La Minière 1961: valeurs moyennes observées sur to vaches au pâturage)

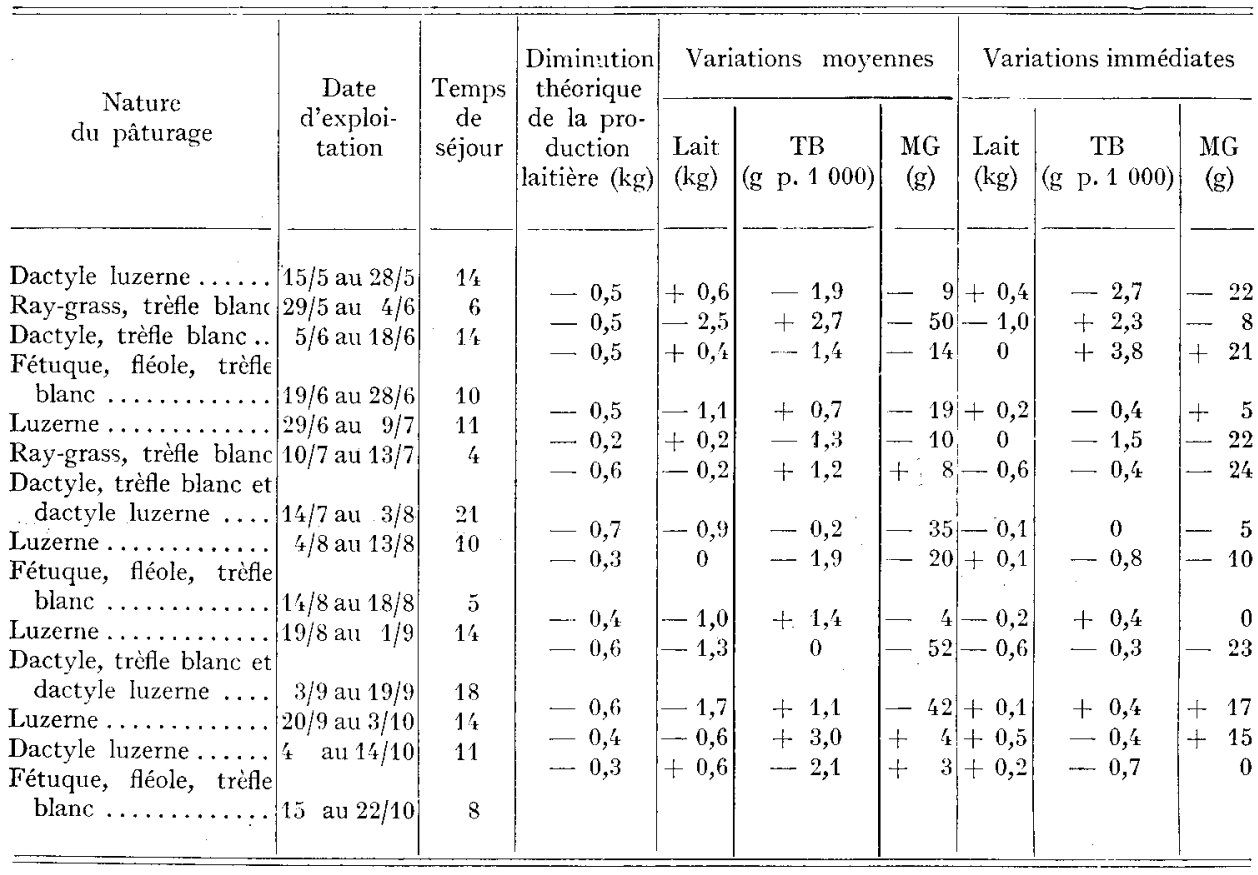

\section{Production laitière.}

A la mise à 1'herbe, la production laitière des deux lots était identique, mais celle du lot alimenté en stabulation a diminué très fortement pendant le premier mois ; aussi le I 5 mai était-elle inférieure de près de $3 \mathrm{~kg}$ de lait par vache à celle du lot au pâturage. Malgré cette différence importante, la production laitière des deux lots a présenté, du I5 mai au I er septembre, des variations semblables à la fois entre les prairies et à l'intérieur des prairies (fig. 7).

La production laitière a peu varié au moment des changements de prairies (tableaux I2 et I3), aussi n'avons-nous reporté sur le graphique que les valeurs enregistrées au cours du séjour sur chaque prairie. Comme les années précédentes, la 


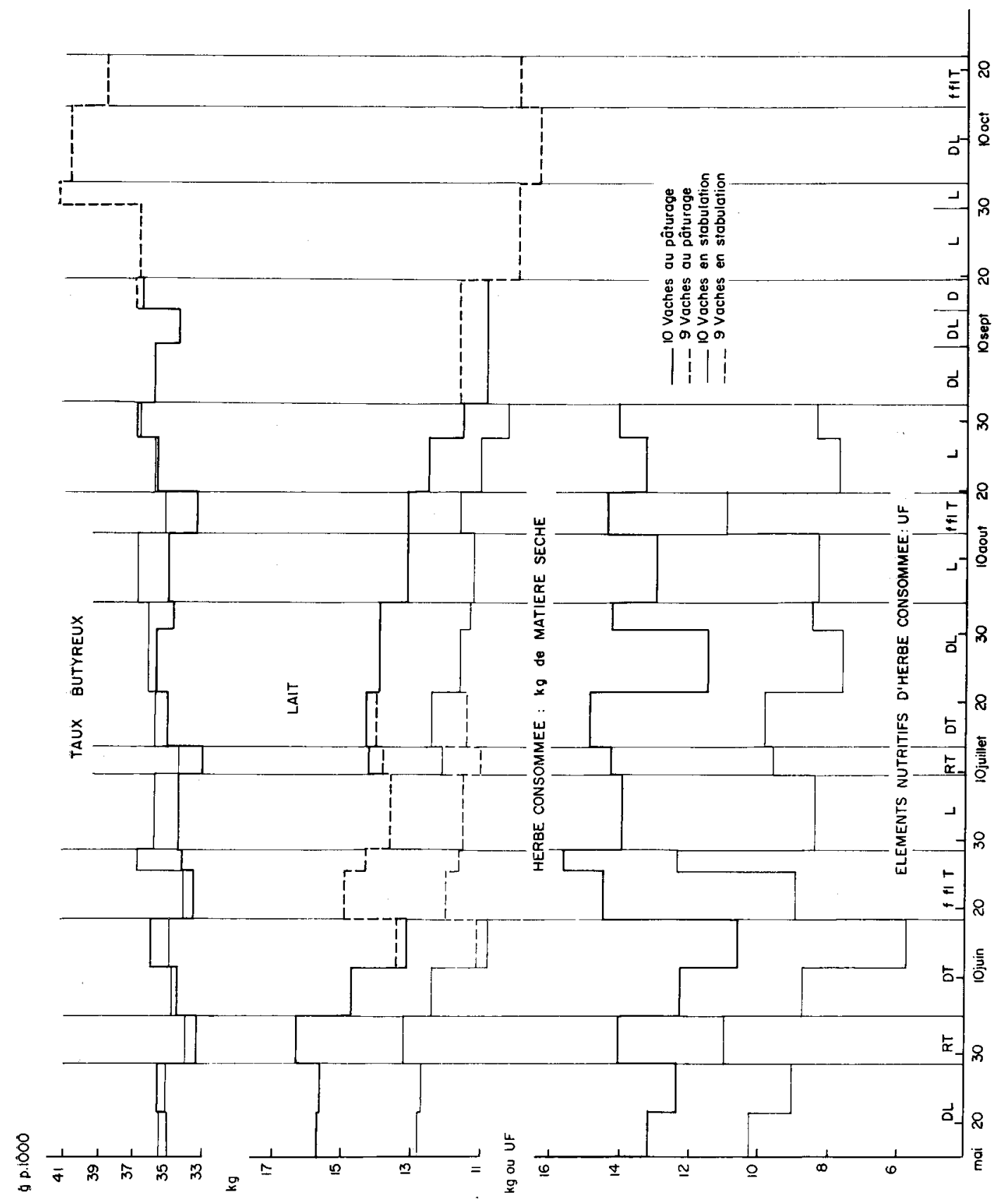

FIG. 7. - Influence de la nature du pâturage sur l'évolution des valeurs moyennes observées au cours du séjour sur différentes prairies pour le lait et le taux butyreux et pour les quantités de matière sèche el d'éléments nutritifs d'herbe consommées (moyenne de ro vaches au pâturage et de ro vaches en stabulation - La Minière, I962).

RT : Ray-grass-Trèfle blanc;

DT : Dactyle-Trèfle blanc;

f fl T : Fétuque des prés-Fléole-Trèfle blanc;

DL : Dactyle-Luzerne ;

L : Luzerne. 


\section{TABLEAU I3}

Variations de la production laitière, du taux bulyreux el de la production de matières grasses en fonction de la nature du pâturage

(La Minière Ig6I : valeurs movennes observées sur ıo vaches en stabulation)

\begin{tabular}{|c|c|c|c|c|c|c|c|c|c|}
\hline \multirow{2}{*}{$\begin{array}{c}\text { Nature } \\
\text { du pâturage }\end{array}$} & \multirow{2}{*}{$\begin{array}{c}\text { Date } \\
\text { d'exploi- } \\
\text { tation }\end{array}$} & \multirow{2}{*}{$\begin{array}{l}\text { Tempss } \\
\text { de } \\
\text { séjour }\end{array}$} & \multirow{2}{*}{\begin{tabular}{|} 
Diminution \\
théorique \\
de la pro- \\
duction \\
laitière $(\mathrm{kg})$
\end{tabular}} & \multicolumn{3}{|c|}{ Variations moyennes } & \multicolumn{3}{|c|}{ Variations immédiates } \\
\hline & & & & $\begin{array}{l}\text { Iait } \\
(\mathrm{kg})\end{array}$ & $\begin{array}{c}\text { TB } \\
(\mathrm{g} p .1000)\end{array}$ & $\begin{array}{l}\mathrm{MG} \\
(\mathrm{g})\end{array}$ & $\begin{array}{l}\text { Lait } \\
(\mathrm{lgg})\end{array}$ & $\begin{array}{c}\text { TB } \\
(\text { (g p. } 1000)\end{array}$ & $\begin{array}{l}\mathrm{MG} \\
(\mathrm{g})\end{array}$ \\
\hline Dactyle luzerne & $15 / 5$ au $28 / 5$ & 1: & & & & & & & \\
\hline Ray-grass, trefle blanc & $29 / 5$ au $4 / 6$ & i & $-0,4$ & $+0,4$ & $-1,1$ & 0 & $\mid-0,3$ & $-1,9$ & -29 \\
\hline Dactyle, trèfle blanc... & $5 / 6$ all $18 / 6$ & $1 ;$ & $-0,4$ & $-1,7$ & $+1,2$ & -45 & $-0,3$ & $+1,8$ & +17 \\
\hline Fétuque fléole, trèfle & & & 一 $0,{ }^{\prime} t$ & $+0,2$ & $-0,3$ & $+\quad 3$ & $-0,1$ & $+4,3$ & +23 \\
\hline blanc $\ldots \ldots \ldots \ldots$ & $19 / 6$ au $28 / 6$ & 10) & - & $-0,4$ & $+0,9$ & -3 & $+0,4$ & $-1,3$ & \\
\hline Luzerne...$\ldots \ldots \ldots$ & $29 / 6$ au $9 / 7$ & 11 & $-0,3$ & $-0,4$ & $-1,4$ & -30 & $-0,5$ & $-1,2$ & -31 \\
\hline $\begin{array}{l}\text { Ray-grass, trèfle blanc } \\
\text { Dactyle trèfle blanc et }\end{array}$ & $10 / 7$ all $13 / 7$ & 4 & -0.4 & $-0,3$ & $+1,9$ & +11 & $-0,5 \mid$ & 0 & -18 \\
\hline dactyle luzerne ... & $14 / 7$ au $3 / 8$ & 21 & 一 0,6 & $-0,5$ & $+0,4$ & -27 & $-0,1$ & 0 & \\
\hline $\begin{array}{l}\text { Luzeme } \ldots \ldots \ldots \ldots \ldots \\
\text { Fétuque, fléole trèle }\end{array}$ & $1 / 8$ au $13 / 8$ & 10 & $\begin{array}{r}0,0 \\
-0,3\end{array}$ & $-0,4$ & $-1,6$ & +10 & $+0,8$ & $-1,5$ & +12 \\
\hline $\begin{array}{r}\text { blanc } \ldots \ldots \ldots \ldots \ldots \\
\text { Luzerne } . . . . \ldots \ldots \ldots\end{array}$ & 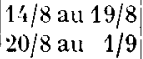 & $\begin{array}{r}6 \\
13\end{array}$ & $-0, x_{1}$ & $-0,8$ & $+0,8$ & -39 & $-0,2$ & $+0,1$ & - 7 \\
\hline
\end{tabular}

production laitière a été plus élevée quand les vaches ont consommé du Ray-grass ou le mélange Fétuque des prés-Fléole que lorsqu'elles ont consommé du Dactyle ou de la Luzerne, mais les différences ont été plus faibles.

Compte tenu de l'avancement de la lactation, la production laitière moyenne a varié par rapport à celle enregistrée au cours des séjours antérieurs sur des prairies de Dactyle ou de Luzerne :

- au pâturage de $+\mathrm{I}, \mathrm{I}$ et $+0,4 \mathrm{~kg}$ de lait par vache et par jour au cours des deux séjours sur les prairies de Ray-grass, et de $+0,9$ et $+0,3 \mathrm{~kg}$ de lait parvache et par jour au cours des deux séjours sur les prairies de Fétuque des prés-Fléole ;

- en stabulation de $+0,8$ et $-0, \mathrm{x} \mathrm{kg}$ de lait par vache et par jour au cours des deux séjours sur les prairies de Ray-grass et de $+0,6$ et $+0,7 \mathrm{~kg}$ de lait par vache et par jour au cours des deux séjours sur les prairies de Fétuques des prés-Fléole.

Inversement, elle a diminué quand les vaches ont quitté les prairies de Raygrass ou de Fétuque des prés-Fléole pour des prairies de Dactyle ou de Luzerne. Elle n'a pas varié quand les vaches sont passées d'une prairie de Dactyle à une prairie de Luzerne.

A partir du I er septembre, la production laitière du lot au pâturage, resté seul en observation, a diminué lors des pâturages successifs de Luzerne et de Dactyle et a augmenté quand les vaches ont pâturé une prairie de Fétuque des prés-Fléole.

Taux butyreux.

Les variations du taux butyreux ont été presque semblables dans les deux groupes.

Le taux butyreux a relativement peu varié au moment des changements de 
prairies, tout au moins si on excepte les premiers, et les variations immédiates ont parfois été en sens contraire des variations moyennes. C'est ainsi que le taux butyreux des deux premiers jours sur une prairie de Fétuque des prés-Fléole a été supérieur de 3,8 et $4,3 \mathrm{~g}$ p. I 000 respectivement au pâturage et en stabulation à calui des deux jours précédents sur une prairie de Dactyle-Trèlle blanc.

Le taux butyreux moyen au cours du séjour sur chaque prairie a, quant à lui, varié dans le même sens que d'habitude mais les amplitudes ont été plus faibles. Il a été systématiquement plus élevé quand les vaches ont consommé du Dactyle ou de la Luzerne et plus faible quand elles ont consommé du Ray-grass ou de la Fétuque-Fléole.

Il n'a pas varié quand les vaches sont passées d'une prairie de Dactyle à une prairie de Luzerne ou vice versa, sauf lors des deux dernières exploitations de ce type de prairies. Les variations alors enregistrées semblent essentiellement dues à l'augmentation du taux butyreux avec la saison et l'avancement de la lactation, les vaches approchant de la période de tarissement.

\section{Production de matières grasses.}

La production de matières grasses a évolué très régulièrement chez les deux lots et elle n'a pas présenté de variations nettes avec la nature du pâturage. On peut donc dire que, contrairement aux années antérieures, l'augmentation du taux butyreux, lorsque les vaches ont consommé du Dactyle ou de la Luzerne, a compensé la diminution de la production laitière qui en est résultée.

\section{Quantité d'éléments nutritifs consommée.}

Les vaches ont consommé beaucoup plus de Ray-grass et de Fétuque-Fléole (respectivement $\mathrm{I}_{4}$, Io et $\mathrm{I} 4,60 \mathrm{~kg}$ de matière sèche par jour et par vache) que de Luzerne et surtout de Dactyle (respectivement 13,65 et I2,55 kg par jour et par vache) (tabeau I4). Comme en I959 et I960, la quantité consommée a varié brusquement au moment des changements de prairies (fig. 7).

Le Ray-grass et le mélange Fétuque des prés-Fléole ont été en moyenne plus digestibles que le Dactyle et surtout que la Luzerne (tableau I4). La différence assez importante, 3 points, entre les coefficients moyens de digestibilité de la matière organique du Ray-grass et de la Fétuque-Fléole est due à la variété de Ray-grass hybride employée (ray-grass Io) qui a épié à chaque exploitation. Le Ray-grass et la Fétuque-Fléole ont cependant eu la même valeur nutritive moyenne $(0,74$ UF au $\mathrm{kg}$ de matière sèche) supérieure de 0,07 et $0, \mathrm{I} 4 \mathrm{UF}$ au $\mathrm{kg}$ à celle du Dactyle et de la Luzerne respectivement.

Le calcul du coefficient moyen de digestibilité de la matière organique a été effectué sur des fourrages qui étaient des repousses pour les ray-grass, les fétuques et les luzernes. Il n'en a pas été de même pour les dactyles, certains étant encore au I 5 mai dans leur premier cycle de végétation. Si l'on ne considère que les repousses de dactyle, leur coefficient moyen de digestibilité de la matière organique a été de 67 p. Ioo.

Le Ray-grass et la Fétuque-Fléole ayant été consommés en plus grande quantité et leur valeur nutritive étant plus élevée, les vaches ont en moyenne consommé chaque jour beaucoup plus d'éléments nutritifs avec ces fourrages (respectivement 


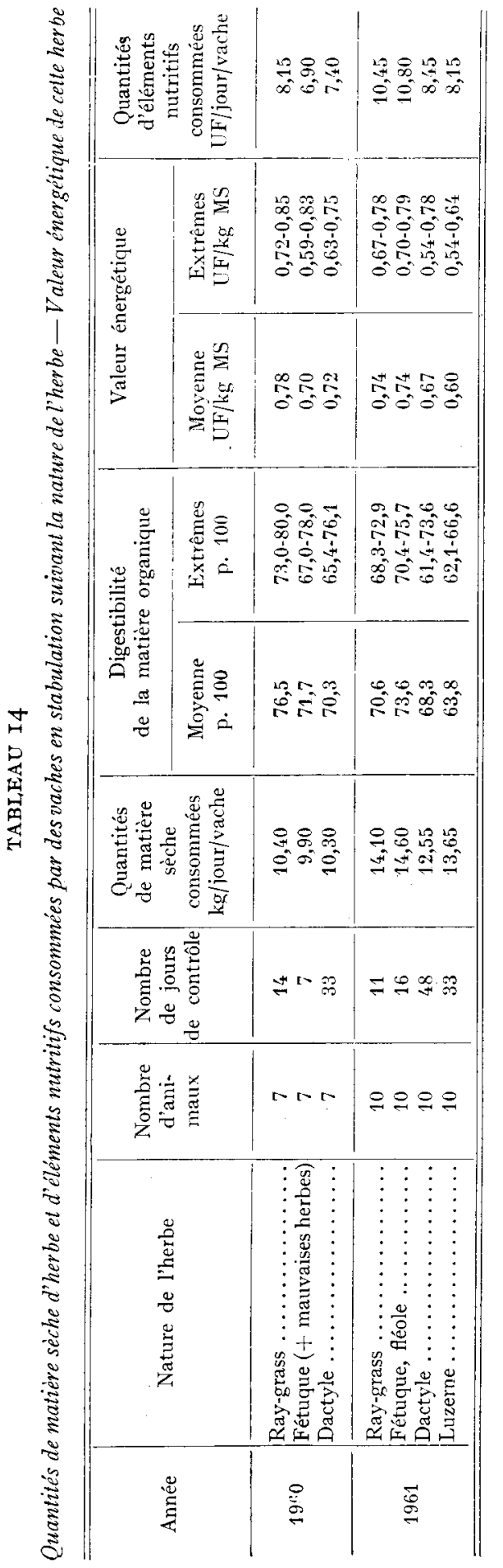

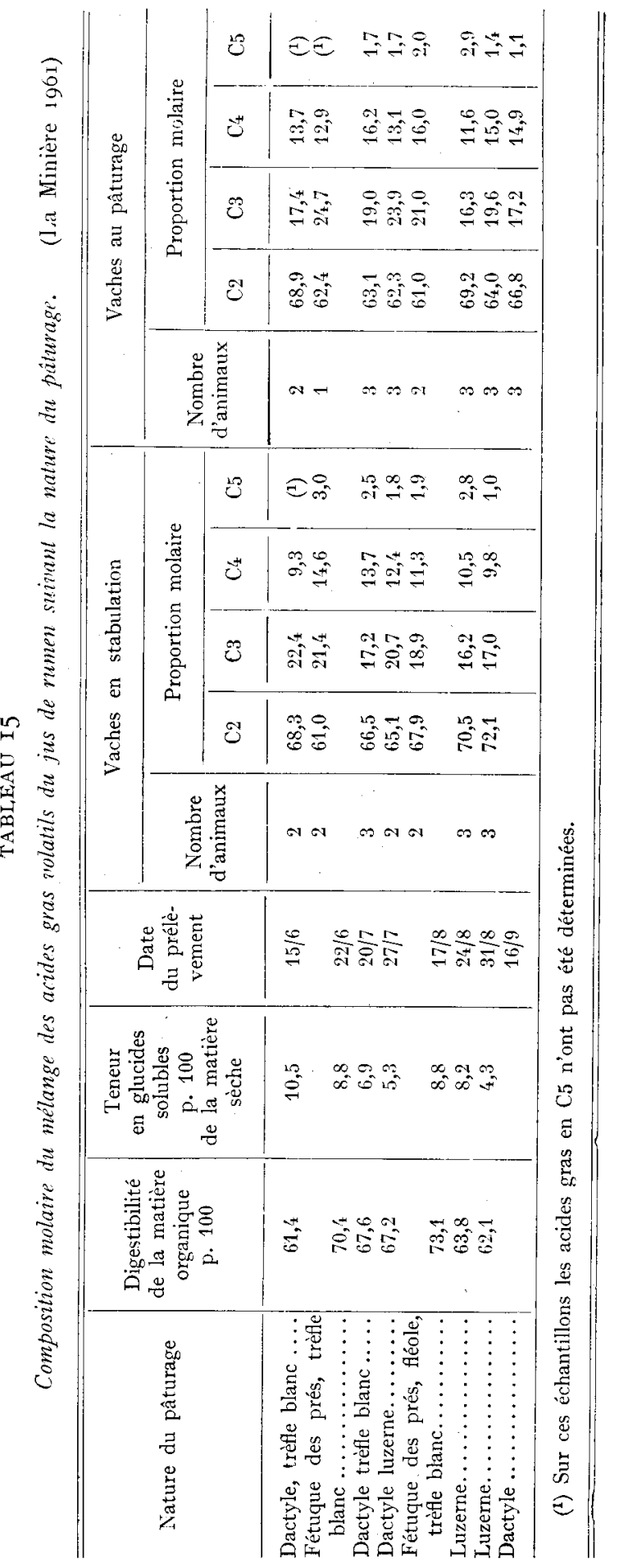


Io,45 et Io,8o UF par vache) qu'avec le Dactyle ou la Luzerne (respectivement 8,45 et 8, I $_{5}$ UF par vache) (tableau I4). La quantité d'éléments nutritifs consommée a donc varié d'une façon importante suivant les prairies (fig. 7).

\section{Composition du mélange des acides volatils du jus de rumen.}

Nous avons constaté des variations relativement importantes mais peu répétables, de la composition du mélange des acides gras volatils du jus de rumen, suivant la nature de l'herbe consommée tant par les vaches au pâturage que par les vaches en stabulation (tableau $\mathrm{I}_{5}$ ).

Le mélange d'acides gras volatils du jus de rumen des vaches qui ont consommé de la Fétuque des prés-Fléole a été systématiquement plus pauvre en acide acétique et plus riche en acide propionique, que celui des vaches qui ont consommé du Dactyle et surtout de la Luzerne. Ce fait a été très bien obsarvé chez les vaches au pâturage mais a été beaucoup moins net chez les vaches en stabulation. D'ailleurs, le mélange des acides gras volatils n'a pas eu la même composition chez les vaches au pâturage et chez les vaches en stabulation. Chez celles-ci, le mélange a presque toujours été plus riche en acide acétique, en particulier quand elles ont consommé de la Luzerne, et il a été plus pauvre soit en acide propionique, soit en acide butyrique.

\section{DISCUSSION}

Au cours de quatre années consécutives dans les deux stations de Jouy et de LA Minière, nous avons constaté que la nature du pâturage avait eu une action nette et reproductible sur la production laitière et sur le taux butyreux; cependant, cette action a eu une amplitude variable, suivant les périodes de l'année, les années et les stations.

La production laitière moyenne pendant les périodes où les vaches ont consommé du Dactyle presque pur, du Dactyle ou de la Fétuque élevée associées à de la Luzerne, a été inférieure à celle enregistrée pendant les périodes où les vaches ont consommé des Ray-grass, de la Fétuque des prés ou de la Fléole associés ou non à du Trèfle blanc. Le taux butyreux moyen a présenté des variations systématiquement inverses : il a été plus faible lorsque les vaches ont consommé des Ray-grass, de la Fétuque des prés, de la Fléole (sauf si ces fourrages étaient exploités l'année du semis), et a augmenté lorsque les vaches ont consommá du Dactyle, de la Fétuque élevée ou de la I uzerne. En général, les variations du taux butyreux n’ont pas été assez importantes pour compenser les variations inverses de la production laitière; la production de matières grasses a donc le plus souvent évolué dans le mêmə sens que la production laitière.

Les variations de la production laitière et du taux butyreux sont apparues immédiatement après le changement de pâturage.

Les variations moyennes ou immédiates de la production et de la composition du lait avec la nature du pâturage, enregistrées à un moment donné, ont toujours eu chez toutes les vaches du lot, des amplitudes presque identiques, indépendantes du niveau de la production laitière. Les vaches produisant peu de lait ont répondu 
aux changements de pâturage avec la même intensité que les vaches en produisant beaucoup.

Il serait imprudent de généraliser ces résultats à toutes les variétés des espèces fourragères étudiées, et à toutes les régions. Ils ont été obtenus dans des conditions bien définies (variétés, milieu, méthodes et exploitation). Nous pensons cependant qu'ils ont une signification générale, car ils ont été obtenus pendant plusieurs années consécutives, avec plusieurs variétés, sous des conditions climatiques très différentes (tableau 3), et dans deux stations aux sols différents ; les prairies de La Minière sont situées sur un plateau de limon relativement sec, celles de Jouy dans une vallée anciennement marécageuse. En outre, d'autres auteurs (CASTLE, I953) (PATERson, I956-I959) (IVIvs, I959) (Boyd \& Jennings, I960), ont eux aussi mis en évidence des variations de la production laitière suivant la nature du pâturage, et observé en particulier une "valeur laitière " plus faible pour les prairies à base de Dactyle, et cela avec d'autres variétés, d'autres modes d'exploitation, et sous d'autres conditions de milieu. En revanche, aucune étude, à notre connaissance, n'avait mis en évidence des variations du taux butyreux avec la nature du pâturage.

Quelles sont les causes possibles de ces variations de la quantité et de la composition du lait suivant la nature de l'herbe consommée? Dans l'état actuel de nos connaissances, elles peuvent être recherchées dans la variation du niveau des apports énergétiques, dans des modifications des processus de fermentation dans le rumen, et peut-être dans certaines propriétés intrinsèques de l'herbe qui peuvent venir amplifier ou modifier les deux actions précédentes.

\section{Variations du niveau des apports énergétiques}

Nous n'avons malheureusement pas pu mesurer la quantité d'éléments nutritifs effectivement consommée par les vaches au pâturage au cours des quatre années étudiées. En revanche, nous l'avons mesurée en I960 et I96I chez les vaches alimentées en stabulation, et nous avons mis en évidence que la production laitière (et a un degré moindre le taux butyreux) des vaches en stabulation, présentait la même évolution et les mêmes variations que celle des vaches au pâturage (fig. 4, 5 et 7 ). Cela nous permet de supposer que les quantités d'éléments nutritifs consommées ont varié de la même façon pour les deux groupes.

Or, il résulte de l'examen des données de I960 et I96r, que la production laitière a présenté les mêmes variations que la quantité de matière organique digestible et d'énergie consommée (fig. 6 et 7). C'est ainsi que cette quantité consommée avec les Ray-grass ou le mélange Fétuque des prés-Fléole, a été supérieure à celle consommée avec le Dactyle ou la Luzerne (tableau I4, fig. 6 et 7). On peut estimer qu'avec les Ray-grass les vaches ont consommé en moyenne $0,75 \mathrm{UF} / \mathrm{jour}$ de plus qu'avec le Dactyle en I960, et respectivement 2,0 et 2,3 UF/jour de plus qu'avec le Dactyle et la Luzerne en Ig6r.

Seul le niveau des apports énergétiques a pu avoir une influence car, les vaches ont toujours eu une quantité suffisante de matières azotées digestibles. Fn effet, la teneur en matières azotées digestibles des fourrages étudiés a été en général relativement élevée; exprimée en grammes de matières azotées digestibles par unité fourragère $(\mathrm{mad} / \mathrm{UF})$, elle a été en moyenne de : 230 et I70 respectivement pour les Ray-grass et le Dactyle en I960 et I40, I30 et 300 respectivement pour le Ray-grass ou le mé- 
lange Fétuque des prés-Fléole, le Dactyle et la Luzerne en I96I. De plus, l'aliment concentré distribué aux vaches en complément de l'herbe avait une teneur de $150 \mathrm{~g}$ de matières azotées digestibles par $\mathrm{kg}$, soit I $80 \mathrm{~g} / \mathrm{UF}$.

Les variations du niveau des apports énergétiques suivant la nature de l'herke consommée, peuvent donc expliquer, au moins en partie, les variations de la production laitière et par là même, de la production de matières grasses ; elles peuvent résulter des variations soit dans la valeur énergétique des fourrages, soit dans la quantité consommée, soit dans les deux à la fois.

\section{Valeur énergétique moindre du Dactyle et de la Luzerne.}

La presque totalité des observations a été faite sur des prairies exploitées après des temps de repos variant de 4 à 7 semaines suivant la croissance de l'herbe, mais à l'intérieur d'un cycle de végétation, la même année et dans la même station, les fourrages étaient d'âge sensiblement égal. Les temps de repos des prairies, différents suivant les années et les stations, permettent d'expliquer la digestibilité plus élevée des fourrages étudiés à Jouy en 1960, année humide, par rapport à celle des fourrages de la Minière en I96I, année sèche. Le fait que la variété de Ray-grass étudiée en I96I a épié à chaque exploitation, a contribué à augmenter encore les différences entre les résultats des deux années.

Quoi qu'il en soit, nous avons constaté qu'en moyenne le coefficient de digestibilité de la matière organique des Ray-grass, a été supérieur de 6,3 et 2,3 points $(3,6$ points si on ne considère que les repousses de Dactyle), à celui du Dactyle respectivement en I960 et 196 I (tableau I4). Ces résultats sont en accord avec ceux enregistrés à Hurley (Mrsson, HaRris et RAYMOND, I960) qui montrent qu'à âge égal le Dactyle $S 37$ a un coefficient de digestibilité inférieur de 5 points à celui des Raygrass $S 24$ et $S 23$. Le coefficient de digestibilité de la Luzerne a été lui-même de 5 points inférieur à celui du Dactyle. On peut donc estimer que les Ray-grass contenaient 0,06 et $0,07 \mathrm{UF} / \mathrm{kg}$ de matière sèche de plus que le Dactyle, respectivement en I960 et I96I, et o, I4 UF de plus que la Luzerne en I96I. La valeur énergétique de la Fétuque des prés a été du même ordre que celle du Ray-grass, la valeur plus faible enregistrée en I 960 étant due à un résultat anormalement bas.

\section{Quantités de matière sèche consommées de Dactyle et de Luzerne moindres.}

Les vaches ont consommé en I960 et I96I systématiquement moins de Dactyle ou de Luzerne que de Ray-grass ou de Fétuque des prés, en $\mathrm{kg}$ de matière sèche (tableau I4). En I959, elles ont aussi consommé moins de Dactyle que de Ray-grass.

Ces résultats sont en accord avec les observations qualitatives enregistrées dans la majorité des essais de "palatibilité " (cf. Ivins, I952) mais non de tous (cf. REID, I95I). Toutes conditions égales par ailleurs, il semble que le Dactyle et, à un moindre degré, la Luzerne ont une " accəptabilité " plus faible que les Ray-grass oula Fétuque des prés, ou diminuant plus rapidement avec 1'âge. Cela peut être dû à certaines caractéristiques chimiques telles qu'une teneur plus faible en glucides solubles, mais surtout à une digestibilité plus faible puisqu'il existe une relation étroite entre le coefficient de digestibilité, et la quantité de matière sèche consommée (BLAXTER et al., I956-I961).

Il semble que l'on puisse généraliser ces résultats aux années antérieures et conclure que, dans les conditions de nos observations, les prairies à base de Dactyle ou 
de I,uzerne ont dî̀ avoir une valeur énergétique de 0,05 à $0,15 \mathrm{UF} / \mathrm{kg}$ de matière sèche inférieure, à celle des prairies à base de Ray-grass ou de Fétuque des prés, et qu'elles ont dû être consommées en moindres quantités.

L'étroite similitude des courbes de production laitière et de taux butyreux des vaches au pâturage et des vaches en stabulation, est un résultat intéressant. Il nous a permis de relier les variations de la production laitière aux variations du niveau des apports énergétiques. Il aurait été très difficile de mettre cette liaison en évidence dans les conditions du pâturage. Cependant il convient de souligner que les quantités consommées par des vaches recevant de l'herbe coupée, ont probablement été différentes de celles consommées au pâturage ; elles ont dû vraisemblablement être plus faibles car les repas ont eu lieu à heure fixe et en temps limité, et l'herbe apportée à l'auge a une composition différente de celle qui est pâturée ; le tri effectué par les animaux a été moins important, en particulier en ce qui concerne le choix des feuilles au détriment des tiges. En revanche, cette méthode doit nous permettre de suivre les variations relatives des quantités d'éléments nutritifs récoltées au pâturage.

\section{Modifications des processus des fermentations dans le rumen}

D'après les divers essais réalisés par plusieurs auteurs il ne semble pas que les variations du niveau des apports énergétiques puissent modifier le taux butyreux des vaches qui reçoivent par ailleurs une quantité suffisante de matières azotées. Ce fait a été établi par de nombreux auteurs (cf. revue bibliographique de JARRIGE et JouRNET, I959). En outre, dans les conditions normales d'alimentation, avec les rations hivernales notamment, il n'y a pas obligatoirement une relation inverse entre la quantité de lait et le taux butyreux. Cette relation n'existe à coup sûr qu'à l'échelle de la lactation ou dans les tous derniers mois de la lactation, lorsque les facteurs physiologiques deviennent la composante principale des, variations journalières du volumeet de la composition du lait (JARRIGE et JOURNE'T, I959). De plus, les variations du niveau des apports énergétiques ne semblent pas pouvoir expliquer totalement les variations extrêmement brutales de la production laitière enregistrées certaines années lors des changements de pâturage.

On sait, depuis que les travaux réalisés aux États-Unis (TyzNick et ALIEN I95I; Shaw, I959), en Australie (Mc Clymont, I959), en Angleterre (Balch et Rowland, I959; RoOK et BAICH, I959; RoOK, I959; $\mathrm{BALCH}_{1}$, I960), que les variations des fermentations du rumen peuvent entraîner des variations (en général immédiates) de la production et de la composition du lait; en particulier, le taux butyreux pourrait varier dans le même sens que le rapport acide acétique/acide propionique.

Or, la nature du pâturage peut entraîner des modifications de la composition du mélange des acides gras volatils du rumen; en particulier, les fourrages ayant une digestibilité élevée (PARks et al., Ig6I ; BlAXTER, I960; ArMstrong, Ig60), ou une teneur élevée en glucides solubles (TulLex et al., rg60; BATH et Rook I96I), tendent à abaisser le pH du rumen et à favoriser la production d'acide propionique au détriment de l'acide acétique. BATH, RoOK et RowLAND (I962) faisant consommer divers fourrages verts par des vaches fistulées, au pâturage ou en stabulation, ont trouvé le pourcentage d'acide acétique le plus faible (associé à un pourcentage d'acide propionique élevé) quand les vaches consommaient du Ray-grass d'Italie 
au stade feuillu, et le pourcentage d'acide acétique le plus élevé (associé à un pourcentage d'acide propionique faible) quand les vaches consommaient soit du Dactyle, soit du Ray-grass anglais âgé.

Nous avons, quant à nous, constaté à la fois des variations de la composition du mélange des acides gras volatils du rumen et des variations du taux butyreux avec la nature du pâturage (L,a Minière, I $96 \mathrm{I}$ ). En particulier lorsque les vaches ont consommé le mélange Fétuque des prés-Fléole, le taux butyreux a été plus faible et le mélange des acides gras du jus de rumen plus pauvre en acide/acétique et plus riche en acide propionique, que lorsqu'elles ont consommé du Dactyle et de la Luzerne. On peut donc supposer que ces variations sont liées, et que la consommation de Dactyle puisse entraîner une augmentation du rapport acide acétique acide propionique et par là, une augmentation du taux butyreux. Malheureusement, nous ne disposons que des résultats de l'année I96I, durant laquelle les variations du taux butyreux ont d'ailleurs été faibles, et cette hypothèse reste à vérifier.

Si elle 1'était, elle nous permettrait d'expliquer les variations du taux butyreux avec la nature du pâturage et, dans une certaine mesure:a) les variations brutales de la production laitière lors du changement de prairies ; b) l'amplitude différente des variations du taux butyreux avec la nature du pâturage, suivant la période de l'année ou suivant les années, puisque la teneur en glucides solubles des fourrages dépend de la saison (WAITE et BOYD, I953) (JARRIGE, I954), et des conditions climatiques (ARCHIBALD, I96I) ; c) le taux butyreux plus élevé chez les vaches consommant de l'herbe en fin de premier cycle que chez celles consommant des jeunes repousses (Jouy, I959) puisque la proportion d'acide acétique dans le mélange des acides gras volatils du jus de rumen, augmente avec l'âge de l'herbe consommée (PARKs et al., I96I; ARMSTRONG, Ig60).

Il n'est, par ailleurs, pas exclu qu'une partie des variations du taux butyreux soit due à des teneurs différentes du mélange d'acides gras en acide butyrique suivant la nature de l'herbe, comme l'ont montré les récents travaux effectués à Reading (N.I.R.D. Report I96I).

\section{Présence de facteurs inconnus}

Dans l'état actuel de nos connaissances, nous ne pouvons pas expliquer pourquoi le Ray-grass et la Fétuque des prés exploités l'année du semis, n'ont pas provoqué une baisse du taux butyreux (Jouy, I960). Il est vrai cependant, que nous n'avons pas déterminé la composition du mélange des acides gras volatils du rumen, quand les vaches ont pâturé ces deux fourrages. En outre, certaines observations ne peuvent être totalement expliquées par les variations du niveau des apports énergétiques, ou les modifications des fermentations dans le rumen ; c'est le cas notamment du caractère souvent brutal des variations lors du changement de pâturage, en particulier celles du taux butyreux, et de l'amplitude identique des variations de la production laitière des vaches, quels que soient leurs niveaux de production.

Par ailleurs, en juin I96I, dans une autre expérience avec des vaches recevant de l'herbe à l'auge, nous avons pu constater une diminution brutale et très importante de la production laitière $(5 \mathrm{~kg}$ de lait par vache en une semaine) qui ne pouvait être expliquée par une diminution des apports énergétiques. L'herbe provenait d'une prairie dégradée de Ray.grass italien Trèfle violet où en deuxième exploitation le Trèfle violet et le Trèfle blanc constituaient la moyenne partie du fourrage. 
Il est done possible qu'une partie des variations de la production et de la composition du lait, puisse provenir de la présence dans l'herbe de facteurs stimulants ou inhibiteurs vis-à-vis de la glande mammaire ou de l'équilibre hormonal de l'animal. On sait par exemple, que l'herbe contient des cestrogènes (cf. revue bibliographique de POPE I954) bien que les teneurs semblent insuffisantes pour modifier la production et la composition du lait (POPE et al., I959).

Les résultats rapportés dans cette étude correspondent à une suite d'observations dont l'interprétation a posé de nombreux problèmes ; en particulier, l'influence de l'avancement de la lactation ou celles des facteurs climatiques sur la production et la composition du lait, n'ont pu être totalement éliminées. Nous avons cependant pu mettre en évidence une action nette de la nature de l'herbe consommée par les vaches, sur la production laitière et le taux butyreux. Les variations du niveau des apports énergétiques et les modifications des processus des fermentations du rumen avec la nature du pâturage, peuvent expliquer de façon satisfaisante, respectivement, les variations de la production laitière, et celles du taux butyreux. L'hypothèse de l'intervention de facteurs stimulants ou inhibiteurs, n'est pourtant pas à rejeter.

Nous nous proposons de continuer cette étude afin de préciser certains points, en particulier les liaisons qui semblent exister entre les variations du taux butyreux et celles de fermentations du rumen, avec la nature du pâturage. Il nous faudra pour cela adopter un dispositif expérimental qui permette d'éliminer l'influence du stade de lactation et des facteurs climatiques, et travailler sur des prairies rigoureusement mono-spécifiques, exploitées en même temps, au même âge et à l'intérieur du même cycle de végétation.

Reşu pour publication en mars 1963

\section{SUMMARY}

THE EFFECTS OF DIFFERENT LEYS ON THE YIELD AND COMPOSITION OF THE MILK OF THE COW

1. Variations in the yield and composition of milk with the type of leys, have been studied during 4 consecutive years (I958-1961), in the two stations of Jouy-en-Josas and La Minière.

This experiment was conducted with 97 cows (table I). The cows were strip-grazed or received every morning in the byre, herbage cut from the same leys. It was carried out under very different climatic conditions (table 3 ). The character of each ley used is given in table 2.

2. The milk produced by each cow was weighed at each milking and the fat content was determined on the weighted composite sample representing a single day for each cow. The quantity of dry matter of the grass consumed each day by the housed cows was individually weighed in I959, 1960 and 1961 and the digestibility of the grass was measured with groups of 5 sheep in 1960 and i 96 I (table i4). In I96I, rumen contents of cows were analysed for individual volatile fatty acids (table I 5 ).

3 . The results obtained each year are given in the following way : a) by a figure showing the daily milk and fat yields, and fat content during the periods spent on the different leys - $b$ ) by a table giving the difference per day and per cow between the average values recorded during the period on two successive leys, and between the average values recorded during the last two days on one ley and the first two days on the following ley.

4. The nature of the ley had a definite and reproductible effect on the yield and composition of milk. When the cows, after consuming ryegrass, meadow fescue, timothy and white clover, ate cocksfoot, tall fescue or lucerne, the milk yield decreased ( 16 times out of 17 outdoors, and 6 times out of 9 indoors) on an average respectively by, I.o and $0.8 \mathrm{~kg}$ milk/day/cow grazing and given 
cut grass indoors, and the fat content increased ( 14 times out of $r 7$ outdoors, and 8 times out of 9 indoors) on an average respectively by, $\mathrm{r} .8$ and $\mathrm{I} .2 \mathrm{~g} \mathrm{p}$. I 000 outdoors and indoors. Inversely, when the cows ate ryegrass, meadow fescue or timothy again the milk yield increased ( 6 times out of $\mathrm{I} 8$ outdoors, and 4 times out of 9 indoors) on an average respectively by 0.6 and $0.2 \mathrm{~kg} \mathrm{milk} / \mathrm{day} /$ cow grazing and given cut grass indoors, and the fat content decreased (16 times out of 18 outdoors, and 7 times out of 9 indoors) on an average respectively by 1.8 and $0.7 \mathrm{~g}$ p. I ooo outdoors and' indoors (fig. I to 7 ) (tables 4,5 and 7 to 13 ).

However, the extent of this action varied with the season, the year studied and the station.

5. The variations in the milk yield and fat content of milk appeared immediately after the change of ley. The variations recorded with cows given cut grass indoors ran parallel with those of cows grazing the same ley (fig. 4, 5 and 7 ). The low-yielding cows reacted to a change of leys in the same way as the high-yielding cows.

6. The average daily intake of grass dry matter consumed by cows given cut herbage and the digestibility of this dry matter varied with the type of grass (table I4) (fig. 6 and 7 ). In I960, the cows ate on an average $8.15 \mathrm{UF} / \mathrm{day} / \mathrm{cow}$ with ryegrass against $7.40 \mathrm{UF} / \mathrm{day} / \mathrm{cow}$ with cokscfoot and in $196 \mathrm{I}, 10.45 \mathrm{UF} /$ day/cow with ryegrass against respectively 8.45 and $8.15 \mathrm{UF} /$ day/cow with coocksfoot and lucerne (UF = feed unit).

7. The causes for variation in the yield and composition of milk with the type of sward are discussed. Variations in the level of energy intake appear to be the main cause of variations in milk yield, and the modifications in the composition of volatile fatty acids produced in the rumen may explain the variation in the fat content of milk. However, the grass may possibly contain inhibitory or stimulatory factors.

\section{RÉFÉRENCES BIBLIOGRAPHIQUES}

Archibald J. G., 196r. Influence of weather on sugar content of forage crops. J. Dairy Sci., 44, 51 I-5 I4.

ARmstrong D. G., ig60. Calorimetric determination of the net energy value of dried S. 23 ryegrass at four stages of growth. Proc. 8th internat. Grassl. Congr., 485-489.

Balch C. C., Rowland S. J., I959. Studies of the secretion of milk of low fat content by cows on diets low in hay and hight in concentrates. vII. The effect of administration of volatile fatty acids to cows giving normal milk and milk of low fat content. J. Dairy Res., 26, I62.

Balch C. C., 1960. Rumen digestion and herbage utilization. Proc. 8th internat. Grassl. Congr., $528-533$.

ВАTH I. H. RоOK J. A. F., I96I. The effect of stage of growth of S. 23 perennial ryegrass on the production of volatile fatty acids in the rumen of the cow. Proc. Nutr. Soc., 20. XV.

Bath I. H. Rook J. A. F. Rowland S. J., 1962. Effects of grazing on the ruminal production of volatile fatty acids in relation to the protein and fat contents of milk. ${ }^{6} 6^{\mathrm{e}}$ Congr. internal. laiterie, $49^{-} 5^{6}$.

Blaxter K. L. Graham N. Mc C. Wainman F. W., 1956. Some observations on the digestibility of food by sheep, and on related problems. Brit. J. Nutr., 10, 69-9r.

Blaxter K. L., r960. The utilization of the energy of grassland products. Proc. 8 ih internat. Grassl. Congr., $479-484$

Blaxter K. L., Wainman F. W. Wilson R. S., ig5r. The regulation of food intake by sheep. Anim. Prod., 3, 5I-6I.

Boyd M., Jennings J. W., ig6o. A comparison of the effects of different swards on the milk yields of dairy cows at Greenmount Agricultural College. The research and experimental Record of the Ministry of Agriculture, Northern Ireland., 9. Part 2, 135-146.

Breirem K., I954. Die Nettoenergie als grundlage der bewertung der futtermittel, in : Nehrivg K., roo jahre Möckern - Die bewertung der futterstoffe und andere probleme der Tiernährung. Berlin, Deutsche Akad. der Landwirtschaftswissenschaften, T II, 97-108.

Castle M. E., 1953. Grassland production and its measurement using the dairy cow. J. Brit. Grassl. Soc., 8, I95-2II.

Demarquilly C., Journet M., I962. Variations de la composition du lait à la mise à l'herbe. I6e Congr. internat. Laiterie, $33^{-48}$.

ElsDen S. R., r946. The application of the silica gal partition chromatogram to the estimation of volatile fatty acids. Biochem. $J ., 40,252-256$.

Friedemann T. E. J., I938. The identification and quantitative determination of volatile alcohols and acids : Biol. Chem., 123, I6r-184.

Ivins J. D., I952. The relative palatibility of herbage plants. J. Brit. Grassl. Soc., 7, 43-54.

IviNs J. D., 1959. The interpretation of animal production data in grassland evaluation. in The measurement of grassland productivity (Proc. Univ. of Nottingham 6th Easter School in Ag. Sci.) Ed. J. D. IvINS - Butterworths Scientific Pubs. 
JAmES A. T., Martin A. J. P., I952. Gas liquid partition chromatography : the separation and micro-estimation of volatile fatty acids form formic acid to dodecanoic acid. Biochem. J., 50, 679-69o.

JARRIGE R., I954. Nature et importance des glucides solubles dans les plantes fourragères en croissance. Conférence Européenne des herbages, 293-298.

JARRIGE R., JourNet M., 1959. Production laitière et pâturage des prairies temporaires. Bull.tech.Inf. $\mathrm{n}^{\circ} \mathrm{I} 45$.

JARRIGE R., JOURNET M., 1959. Influence des facteurs alimentaires et climatiques sur la teneur en matières grasses du lait. Ann. Nutr. Alim., 13, 233-277.

Minson D. J., Raymond W. F., Harris C. E., 1960. The digestibility of grass species and varieties. Proc. 8th internat. Grassl. Congr., 470-474.

National Institute for Research in Dairying. Report 196r. p. go.

Parks P. F., Riewe M. E., Kunkel H. O., Lyman C. M., г96г. The effect of stage of maturity of gulf ryegrass on the intraruminal fatty acid ratios of sheep. (abstract.). J. Anim. Sci., 20, 962.

Paterson R., 1956. The milk stimulating value of grass and its influence on animal health. J. Brit. Grassl. Soc., 11, 93-98.

PATERSON R., I959. Measuring the milk stimulating values of feeds. In the measurement of Grassland productivity. (Proc. Univ. of Nottingham 6th Easter School in Ag. Sci.). Ed. J. D. Ivins. Butterworths Scientific Pubs.

POPE G. S., 1954. The importance of pasture plant oestrogens in the reproduction and lactation of grazing animals. Dairy Sci. Abstr., 16, 344-355.

Pope G. S., Mc Naughton M. J., Jones H. E. H., 1959. Oestrogens in British pasture plants. J.Dairy Res., 26, I96-202.

REID D., 195I. A quantitative method for determining palatibility of pasture plants. J. Brit. Grassl. Soc., 6, I87-195.

Rook J. A. F., 1959. Milk composition in relation to rumen metabolism. Proc. Nutr. Soc., 18, i 7 .

Rook J. A. F., BALCH C. C., 1959. The effects of intraruminal infusions of acetic and propionic acids on the yield and composition of the milk of the cow. Proc. Nutr. Soc., 18, XXXIV.

SHAw J. C., 1959. Nutritional Physiology of the rumen : A new approach to rumen nutrition. Feedstuffs, 31, $18-22$.

Tilley J. M. A., Deriaz R. E., Terry R. A., I960. The in vitro measurement of herbage digestibility and assessment of nutritive value. Proc. Sth internat. Grassl. Congr., 533-537.

Tyznich W., AlLEN N. N., 195I. The relation of roughage intake to the fat content of the milk and the level of fatty acids in the rumen. J. Dairy Sci., 34, 493.

Vallance W. S., Mc Clymont G. L., 1959. Depression in percentage of milk fat by parental glucose infusion and glycerol feeding. Nature Lond., 183, 466-467.

WAITE R., BoyD J., I953. The water soluble carbohydrates of grasses. II. Grasses cut at grazing height several times durint the growing season. J. Sci. Food Agric., 4, 257.

ZELTER Z., 1958. Azote urẻique et activité bactérienne in vitro au niveau du rumen. r. Effet de l'urée sur la digestion des glucides d'une paille de blé et d'une farine de luzerne déshydratée. Ann. Zootech., 7, 1 73-183. 